Notfall Rettungsmed 2015 · 18:984-1002

DOI 10.1007/s10049-015-0084-y

Online publiziert: 14. Oktober 2015

(C) European Resuscitation Council (ERC),

German Resuscitation Council (GRC), Austrian

Resuscitation Council (ARC) 2015

N.I. Nikolaou' • H.R. Arntz ${ }^{2}$ - A. Bellou ${ }^{3,4} \cdot$ F. Beygui ${ }^{5} \cdot$ L.L. Bossaert ${ }^{6} \cdot$ A. Cariou $^{7}$

${ }^{1}$ Cardiology Department, Konstantopouleio General Hospital, Athens, Griechenland

2 Department Emergency Medicine, Charité, University Medicine Berlin, Campus Benjamin Franklin, Berlin, Deutschland

${ }^{3}$ University of Rennes, Rennes, Frankreich

${ }^{4}$ Department of Emergency Medicine Beth Israel Deaconnes Medical Center Harvard Medical School, Boston, USA

${ }^{5}$ Interventional Cardiology Unit Caen University Hospital, Caen, Frankreich

${ }^{6}$ Department of Medicine and Health Sciences, University of Antwerp, Antwerp, Belgien

${ }^{7}$ Medical Intensive Care Unit, Cochin University Hospital (APHP) \& Paris Descartes University, Paris, Frankreich

\title{
Das initiale Management des akuten Koronarsyndroms
}

\section{Kapitel 8 der Leitlinien zur Reanimation 2015 des European Resuscitation Council}

\section{Zusammenfassung der wesentlichen Änderungen seit den Leitlinien 2010 \\ Zusammenfassung der neuen Behandlungsempfehlungen}

Das Folgende ist eine Zusammenfassung der wichtigsten Neuerungen, die die Empfehlungen für Diagnostik und Behandlung des akuten Koronarsyndroms (ACS) seit den ERC Guidelines 2010 betreffen.

\section{Diagnostische Maßnahmen bei ACS}

- Die prähospitale Registrierung eines 12-Ableitungs-Elektrokardiogramms (EKG) bei Patienten mit Verdacht auf einen ST-Strecken-Hebungs-Infarkt (STEMI) beschleunigt nicht nur die prä- bzw. innerhospitale Reperfusion, sondern vermindert auch die Sterblichkeit. Sie wird daher sowohl für Patienten mit geplanter primärer perkutaner Koronarintervention (PPCI) als auch für Patienten, die eine Fibrinolyse erhalten, empfohlen.

zu diesem Kapitel hat beigetragen: Nicolas Danchin, Department of Cardiology, Hôpital Européen Georges Pompidou, Paris, France
- Die STEMI-EKG-Interpretation durch Nichtärzte mit oder ohne Unterstützung durch Computeralgorithmen wird angeregt, wenn eine angemessene diagnostische Qualität durch sorgfältig geführte Qualitätssicherungsprogramme aufrechterhalten werden kann.

- Die prähospitale Aktivierung des Herzkatheterlabors kann nicht nur Behandlungsverzögerungen reduzieren, sondern auch die Patientensterblichkeit verringern.

- Ein negativer Test kardialer „High-Sensitivity“-Troponine (hs-cTn) bei initialer Untersuchung des Patienten reicht als alleinige Messgröße zum Ausschluss eines ACS nicht aus, kann jedoch bei Patienten mit sehr niedrigem Riskoprofil eine frühe Entlassung rechtfertigen.

\section{Therapeutische} Maßnahmen bei ACS

- Bei Patienten mit STEMI-Verdacht kann die Gabe von Adenosindiphosphat(ADP)-Rezeptor-Antagonisten (Clopidogrel, Ticagrelor bzw. mit bestimmten Einschränkungen Prasugrel) prähospital oder in der Notaufnahme bei geplanter PPCI erwogen werden.
- Unfraktioniertes Heparin (UFH) kann entweder schon prähospital oder intrahospital bei Patienten mit STEMI und geplanter PPCI gegeben werden.

- Enoxaparin kann beim STEMI als Alternative zu UFH prähospital gegeben werden.

- Patienten mit akutem Brustschmerz bei vemutetem ACS brauchen keinen zusätzlichen Sauerstoff, sofern sie nicht Zeichen der Hypoxie, Atemnot oder Herzinsuffizienz aufweisen.

Wahl der Reperfusionstherapie bei STEMI

Die Wahl der Reperfusionstherapie wurde unter verschiedenen möglichen, lokalen Bedingungen betrachtet:

- Für den Fall, dass die Fibrinolyse die geplante Behandlungsstrategie ist, soll beim STEMI die prähospitale der intrahospitalen Fibrinolyse vorgezogen werden, wenn die Transportzeiten $>30$ min sind und das Rettungsdienstpersonal gut ausgebildet ist.

- Dort, wo PCI-Kapazitäten vorgehalten werden und verfügbar sind, wird die direkte Auswahl geeigneter Patienten und der Transport zur PCI gegenüber der Fibrinolyse bevorzugt. 


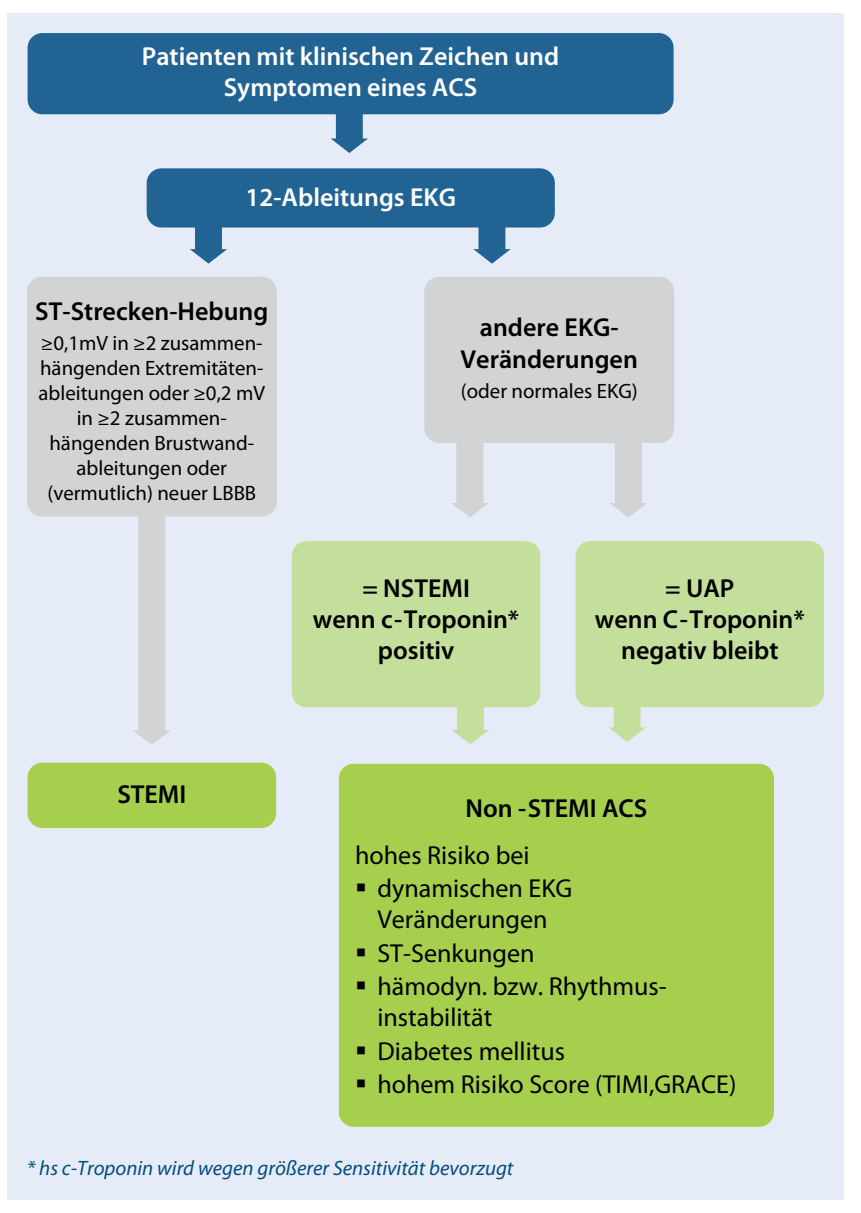

einer koronaren Ursache des Kreislaufstillstands zu denken.

\section{Einführung}

Die Inzidenz des akuten ST-StreckenHebungs-Infarkts (STEMI) sinkt in vielen europäischen Ländern [1]; die Inzidenz des ACS ohne ST-Strecken-Hebung (non-STEMI-ACS) jedoch steigt [2]. Obwohl die Krankenhaussterblichkeit bei STEMI durch die moderne Reperfusionstherapie und eine verbesserte Sekundärprophylaxe signifikant gesenkt werden konnte, bleibt die 28-Tage-Gesamtsterblichkeit anscheinend unverändert, da ca. zwei Drittel derjenigen, die vor Erreichen des Krankenhauses versterben, durch ischämiegetriggerte tödliche Arrhythmien sterben [3]. Daher ist der beste Weg, das Überleben einer Ischämie-Attacke zu verbessern, die Verzögerungszeit vom Symptombeginn bis zum medizinischen Erstkontakt und zur gezielten Behandlung in der frühen Prähospitalphase zu verkürzen.

Der Begriff „akutes Koronarsyndrom" (ACS) umfasst drei unterschiedliche Formen der Akutmanifestation der koronaren Herzkrankheit ( Abb. 1): den ST-Strecken-Hebungs-Infarkt (STEMI), den Infarkt ohne ST-Strecken-Hebung (non-STEMI) und die instabile Angina pectoris (UAP). Der non-STEMI und die UAP werden gewöhnlich unter dem Begriff non-STEMI-ACS zusammengefasst. Der gemeinsame pathophysiologische Hintergrund des ACS ist eine rupturierte oder erodierte atherosklerotische Plaque [4]. Elektrokardiographische (EKG) Charakteristika (Vorhandensein bzw. Fehlen von ST-Strecken-Hebungen) ermöglichen es, zwischen dem STEMI und dem non-STEMI-ACS zu unterscheiden. Letzteres kann mit ST-Strecken-Senkungen, unspezifischen ST-Segment-Abnormalitäten oder sogar mit einem normalen EKG einhergehen. In Abwesenheit von ST-Strecken-Hebungen gilt der Anstieg in der Plasmakonzentration kardialer Biomarker, insbesondere Troponin T oder I, als der spezifischste Marker zum Nachweis eines non-STEMI bei Myokardzellnekrosen. 
Akute Koronarsyndrome sind die häufigste Ursache für zum plötzlichen Herztod führende maligne Arrhythmien. Die Therapieziele sind, akute, lebensbedrohliche Zustände wie Kammerflimmern (VF) oder extreme Bradykardien zu behandeln, die linksventrikuläre Funktion aufrechtzuerhalten sowie der Herzinsuffizienz durch Minimierung des Myokardschadens vorzubeugen. Die aktuellen Leitlinien betonen die Bedeutung der ersten Stunden nach Symtombeginn. Die Therapie außerhalb des Krankenhauses und die Initialtherapie in der Notfallaufnahme (ED) können je nach örtlichen Möglichkeiten, Ressourcen und Regelungen variieren. Die Daten, die die Behandlung außerhalb des Krankenhauses unterstützen, sind oft von Studien zur Initialbehandlung nach Krankenhausaufnahme abgeleitet. Es gibt nur wenige qualitativ hochwertige Studien zur Behandlung außerhalb des Krankenhauses. Umfassende Leitlinien zur Diagnostik und Behandlung des ACS mit und ohne ST-StreckenHebung wurden von der European Society of Cardiology und das The American College of Cardiology/American Heart Association publiziert. Diese aktuellen Empfehlungen stehen in Übereinstimmung mit diesen Leitlinien $[5,6]$.

\section{Diagnose und Risikos- tratifizierung bei ACS}

\section{Zeichen und Symptome des ACS}

Typischerweise zeigt sich das ACS mit Symptomen wie ausstrahlendem Brustschmerz, Luftnot und Schwitzen. Jedoch können atypische Symptome oder ungewöhnliche Anzeichen bei älteren Patienten, Frauen und Diabetikern auftreten. Keines der Zeichen und Symptome eines ACS sichert alleine die Diagnose eines ACS. Schmerzlinderung nach Nitroglyceringabe kann in die Irre führen und wird nicht als diagnostischer Test empfohlen [7]. Die Symptomatik kann bei Patienten mit STEMI intensiver sein und länger anhalten, ist aber nicht verlässlich um zwischen einem STEMI und einem non-STEMI-ACS zu unterscheiden [5, 8-10].

Die Vorgeschichte des Patienten soll während des Erstkontakts mit medizinischem Personal sorgfältig erhoben wer- den. Sie kann den ersten Hinweis für das Vorhandensein eines ACS geben, weitergehende Untersuchungen auslösen und in Kombination mit Informationen aus weiteren diagnostischen Tests helfen, die klinische Einordnung und therapeutische Entscheidungen bereits in der Prähospitalphase bzw. der Notfallaufnahme (Emergency Department, ED) zu treffen.

Das klinische Erkennen eines ACS ist eine Herausforderung, die unterstreicht, dass das Training der Notfallteams einschließlich Leitstellenmitarbeitern, Ärzten und nicht ärztlichen Mitarbeitern je nach Art des Rettungssystems von grundlegender Bedeutung ist. Klinische Ablaufprotokolle werden nachhaltig empfohlen und sollen für Notfallteams, die prähospital oder in der Notfallaufnahme arbeiten, bereitstehen.

\section{Das 12-Ableitungs-EKG}

Ein 12-Ableitungs-EKG ist die nimmt die Schlüsselrolle für die Beurteilung eines ACS ein. Im Fall eines STEMI zeigt sie die Notwendigkeit einer sofortigen Reperfusionstherapie an (primäre perkutane Koronarintervention (PPCI) oder prähospitale Fibrinolyse). Wenn der Verdacht auf ein ACS besteht, soll ein Ausdruck eines 12-Ableitungs-EKG so schnell wie möglich beim ersten $\mathrm{Pa}$ tientenkontakt abgeleitet und interpretiert werden, um eine frühzeitige Diagnose und Einordnung des Patienten zu zu ermöglichen $[6,8,10] .{ }^{1}$ Typischerweise liegt ein STEMI vor, wenn die ST-Elevation, gemessen am J-Punkt, die Voltage-Kriterien in Abwesenheit einer linksventrikulären Hypertrophie bzw. eines Linksschenkelblocks („left bundle branch block“, LBBB) vorliegt [5]. Bei Patienten mit klinischem Verdacht auf eine fortschreitende Myokardischämie mit einem neuen oder vermutlich neuen LBBB soll eine Reperfusionstherapie - vorzugsweise eine primäre PCI (PPCI) - sofort ins Auge gefasst werden. Eine ventrikuläre Schrittmacherstimulation kann das Vorhandensein eines entstehenden Infarkts im EKG verschleiern und eine dringliche Angiographie er-

\footnotetext{
1 Wegen der Gefahr von TF und VT darf darüber aber das EKG-Monitoring nicht vergessen werden.
}

fordern, um die Diagnose zu sichern und die Therapie einzuleiten.

Rechtspräkordiale Ableitungen sollen bei allen Patienten mit inferiorem Infarkt registriert werden, um einen rechtsventrikulären Infarkt aufzudecken. Isolierte STSenkungen $\geq 0,05 \mathrm{mV}$ in den Ableitungen $\mathrm{V} 1-\mathrm{V} 3$ weisen auf einen STEMI im inferobasalen Herzmuskel hin, der durch STSegment-Hebungen in den Ableitungen V7-V9 bestätigt werden kann. Das prähospital oder in der Notfallaufnahme registrierte EKG führt zu wertvollen diagnostischen Informationen, wenn es durch trainiertes medizinisches Personal ausgewertet wird.

Die Registrierung eines prähospitalen EKG ermöglicht die vorzeitige Anmeldung eines Patienten im Zielkrankenhaus und beschleunigt die Entscheidungen nach Eintreffen im Krankenhaus: In zahlreichen Studien wurde mithilfe des prähospitalen 12-Kanal-EKG die Zeit zwischen Krankenhausaufnahme und dem Beginn der Reperfusionstherapie um 10-60 min verkürzt. Dies führt zu einer früher erreichten Reperfusion und einer höheren Überlebensrate der Patienten sowohl bei PCI als auch bei Fibrinolyse [11-19].

Trainiertes Rettungsdienstpersonal (Notärzte, Rettungsassistenten/Notfallsanitäter und Krankenschwestern) kann einen STEMI, definiert als ST-Hebung $\geq 0,1 \mathrm{mV}$ in mindestens zwei zusammenhängenden Extremitätenableitungen bzw. $\geq 0,2 \mathrm{mV}$ in mindestens zwei zusammenhängenden Brustwandableitungen, mit hoher Spezifität und Sensitivität erkennen, vergleichbar mit der Genauigkeit im Krankenhaus [20, 21]. Es ist deshalb sinnvoll, dass Rettungsassistenten, Notfallsanitäter und Krankenschwestern ohne unmittelbare ärztliche Beratung in der STEMI-Diagnostik geschult werden, sofern dies von einer strikten Qualitätssicherung begleitet wird.

Falls eine Interpretation des prähospitalen EKG vor Ort nicht möglich ist, ist eine computergestützte Auswertung [22, 23] oder eine Funkübertragung des EKG sinnvoll [14, 22-29]. Die Registrierung und Übertragung eines EKG in diagnostischer Qualität in das Krankenhaus benötigt üblicherweise weniger als $5 \mathrm{~min}$. Der Einsatz einer computergestützten Aus- 
wertung des EKG bei Patienten mit ACSVerdacht kann die Spezifität einer STEMI-Diagnose steigern, speziell bei Klinikern, die in der EKG-Auswertung unerfahren sind. Der Nutzen einer computergestützten Interpretation ist jedoch abhängig von einer fehlerfreien Registrierung. Fehlerhafte Registrierungen können unerfahrene EKG-Befunder in die Irre führen. Insofern soll die computergestützte EKG-Auswertung die Befundung durch einen erfahrenen Befunder nicht ersetzen, sondern als Ergänzung angesehen werden.

\section{Biomarker, Regeln für die frühzeitige Entlassung und Protokolle zur Beobachtung von Brustschmerzpatienten}

Beim Fehlen von ST-Hebungen im EKG charakterisieren eine verdächtige Vorgeschichte und erhöhte Konzentrationen von Biomarkern (Troponine, CK und CKMB) den non-STEMI und unterscheiden ihn so einerseits vom STEMI und andererseits von der instabilen Angina pectoris. Die Messung eines herzspezifischen Troponins wird routinemäßig wegen der höheren Sensitivität und Spezifität genutzt. Erhöhte Troponin-Werte sind besonders hilfreich bei der Identifikation von Patienten mit erhöhtem Risiko für unerwünschte Ereignisse [30, 31].

Um die Resultate der Biomarkermessung optimal zu nutzen, sollen Kliniker mit der Sensitivität, der Präzision und den Klinik-spezifischen Normalwerten ebenso vertraut sein wie mit der Freisetzungsund Abbaukinetik. Es wurden hochsensitive (ultrasensitive) Tests für kardiale Troponine entwickelt. Sie können die Sensitivität erhöhen und die Diagnose eines Infarkts bei Patienten mit Symptomen einer kardialen Ischämie beschleunigen [32].

Die Bestimmung kardialer Biomarker soll Bestandteil der initialen Beurteilung aller Patienten sein, die sich in der Notfallambulanz mit Symptomen vorstellen, die auf eine kardiale Ischämie hindeuten. Jedoch behindert die verzögerte Freisetzung der Biomarker aus geschädigtem Myokard ihren Nutzen für die Diagnose eines Myokardinfarkts in den ersten Stunden nach Symptombeginn. Bei Patienten, die sich innerhalb der ersten $6 \mathrm{~h}$ nach Symptombeginn vorstellen und einen negativen initialen Troponintest aufweisen, sollen die Biomarker erneut nach zwei bis drei und noch einmal $6 \mathrm{~h}$ später (bei hs-cTn-Test, $12 \mathrm{~h}$ bei Standardtroponintest) gemessen werden. Die Mehrheit der Patienten mit möglichem ACS hat diese Erkrankung nicht, insofern stellt die Identifikation derjenigen, die ein ACS haben, eine schwierige Aufgabe dar. Jüngere Studien berichten über eine Häufigkeit nicht erkannter ACS-Diagnosen von bis zu 3,5\% mit konsekutiv erheblicher Morbidität und Mortalität [33-35].

Mit der Einführung der hochsensitiven (hs) Tests, sind mehrere hs-cTn-basierte Entscheidungspfade mit dem Ziel beschleunigter Entscheidungen geprüft worden, die zu einer wachsenden Zahl von diagnostischen Algorithmen in den Notaufnahmen geführt haben. Diese umfassen das EKG, Troponine und den TIMI-Risiko-Score (TIMI Thrombolysis in Acute Myocardial Infarction). Daten aus großen multizentrischen Beobachtungsstudien zeigen hervorragende Ergebnisse für Protokolle zum Ausschluss der ACS-Diagnose innerhalb von $2 \mathrm{~h}$. In diesen wurden die hs-cTn-Werte mit klinischen Informationen kombiniert. Es existieren aber auch 1-h-Ausschluss- bzw. Einschluss-Protokolle für ein ACS, die allein auf hs-cTn-Werten beruhen [36-39].

Es wird nicht empfohlen, hochsensitive Tests auf kardiales Troponin, initial und nach $2 \mathrm{~h}$ gemessen, als alleinige Grundlage zu nehmen, um die Diagnose eines ACS mit einer MACE-Rate (MACE wesentliche unerwünschte kardiale Ereignisse) $<1 \%$ auszuschließen [40]. Initiale und nach $2 \mathrm{~h}$ gemessene negative hscTn-Werte, können zusammen mit einer Stratifizierung in eine Gruppe mit geringem Risiko (TIMI-Score 0 oder 1 ) zum Ausschluss eines ACS eingesetzt werden. Negative Werte für kardiales Troponin T oder I initial und nach 3-6 h können, zusammen mit der Zuordnung zu einer Gruppe mit sehr geringem Risiko (Vancouver Score 0 oder North American CP Score 0 und Alter $<50$ Jahre), zum Ausschluss eines ACS eingesetzt werden.

Es gibt keine Daten, die den alleinigen Einsatz von „Point-of-Care“(POCT)-Troponintests in der Prähospitalphase als Primärtest unterstützen, um Patienten zu be- urteilen, die ACS-verdächtige Symptome haben [32]. In der Notfallaufnahme kann der Einsatz von POCT-Troponintests helfen, die Zeit zur definitiven Behandlung und die Aufenthaltsdauer in der Notfallaufnahme zu verkürzen [41]. Solange nicht weitere kontrollierte Studien durchgeführt sind, sollen andere Serumtests für die Diagnose und das Vorgehen bei Patienten nicht als initial geeignet angesehen werden, die sich mit ACS-Symptomen vorstellen [42-44].

Es wurden Scores zur Risikobeurteilung und klinische Voraussage-Algorithmen entwickelt, die die Vorgeschichte, die körperlichen Untersuchung, das EKG und kardiale Troponine nutzen, um Patienten mit ACS zu identifizieren, die ein erhöhtes Risiko für einen ungünstigen Verlauf haben. Sowohl detaillierte Befundabgrenzung als auch genaue Eichung sind notwendig zur Berechnung einer Risikovoraussage. Kliniker müssen wissen, welche ACS-Patienten das höchste Risiko tragen, sodass diese vorrangig einer frühzeitigeren und „aggressiveren“ Behandlung zugeführt werden können. Aber sie sollen auch das absolute Risiko kennen, um den Patienten die Gefahren und Vorteile verschiedener Behandlungsoptionen zu erklären und sie bei der Entscheidung unter sinnvoller, vernünftiger Nutzen-RisikoAbwägung zu unterstützen. Das „Global Registry of Acute Coronary Events“ (GRACE-Register) und der TIMI-RisikoScore werden am häufigsten benutzt. In einer kürzlich durchgeführten Metaanalyse waren der TIMI- und der GRACERisiko-Score die einzigen, die sich unter verschiedenen klinischen Bedingungen bewährt haben, wobei der GRACE-Score mit einer AUC (,area under the curve") von rund 0,85 besser abschnitt [45].

Der GRACE-Score identifizierte mit großer Sensitivität und hohem negativem Vorhersagewert eine beträchtliche Gruppe, die potenziell für eine sichere frühe Entlassung aus der Notfallambulanz und für weitere ambulante Betreuung geeignet war. Die Komplexität dieses Instruments begrenzt allerdings seine Anwendbarkeit $[46,47]$. Es dürfte schwierig sein, die genannten Scores im prähospitalen Umfeld, in dem Laborparameter (Biomarker, Kreatinin) nicht verfügbar sind, einzusetzen. Dies ist vermutlich der Grund 
dafür, dass die prähospitalen Aspekte der Versorgung von non-STEMI-Patienten wenig Aufmerksamkeit auf sich gezogen haben, trotz der Häufigkeit und dem wesentlichen Beitrag des non-STEMIACS und seiner herausragenden Bedeutung für die Sterblichkeit am Myokardinfarkt. Ob die Einführung eines regionalen Programms zur frühen prähospitalen Risikostratifizierung, die Einführung evidenzbasierter Versorgung und eine frühzeitige invasive Strategie für Patienten mit mäßigem oder erhöhtem Risiko den Verlauf besser machen wird, bedarf noch der Untersuchung [48]. Die neue Version des GRACE-Risiko-Scores (GRACE 2.0) setzt nichtlineare Funktionen ein und scheint genauer zu sein als die Originalversion. Er liegt jetzt validiert für einen längeren Prognosezeitraum (bis zu 1 Jahr und 3 Jahren) vor. Mit Parametern, mit denen der Ersatz von Kreatininwerten und der Killip-Klasse möglich ist, wird GRACE 2.0 eine Risikostratifizierung beim ersten Kontakt zum Patienten ermöglichen, egal, wo die Behandlung begonnen wird [49].

Bei Patienten mit ACS-Verdacht können selbst eine unauffällige Vorgeschichte und normale körperliche Untersuchungsergebnisse sowie negative initiale Biomarker und ein negatives initiales EKG zum verlässlichen Ausschluss eines ACS herangezogen werden. Deshalb ist eine Weiterbeobachtungsperiode unerlässlich, um eine definitive Diagnose zu stellen und Therapieentscheidungen zu fällen.

Protokolle zur Beobachtung von Brustschmerzpatienten sind Hilfsmittel zur raschen Beurteilung von Patienten mit ACS-Verdacht. Sie sollen generell die Vorgeschichte und die körperliche Untersuchung umfassen, gefolgt von einer Nachbeobachtungsperiode, während der serielle EKG-Registrierungen und Messungen kardialer Marker durchgeführt werden. Die Beurteilung des Patienten soll ergänzt werden, entweder durch eine nichtinvasive Methode zur Erfassung einer krankhaften Koronaranatomie oder einen Provokationstest zum Nachweis einer induzierbaren Myokardischämie einige Zeit nach Ausschluss eines Infarkts. Die Protokolle können benutzt werden, um die Identifikation derjenigen Patienten zu verbessern, die einer stationären Aufnahme bedürfen, oder solcher, die weitere Diagnos- tik brauchen unter Aufrechterhaltung der Patientensicherheit bei gleichzeitiger Verkürzung der Aufenthaltsdauer und Reduzierung der Kosten [50].

Für Patienten, die in die Notfallambulanz mit einem ACS-verdächtigen Befund aufgenommen werden, aber initial normale Untersuchungsergebnisse aufweisen, können Einrichtungen zur Beobachtung von Brustschmerzpatienten („Chest Pain Units") eine sichere und effektive Strategie zur Patientenbeurteilung sein. Der potenzielle diagnostische und therapeutische Zugewinn durch Provokationstests wird eine zunehmend zentrale Bedeutung für die Bestimmung der Nützlichkeit solcher Tests bei Brustschmerzpatienten mit niedrigem und mittlerem Risiko spielen, die im Rahmen beschleunigter Protokolle beurteilt werden sollen. Multizentrische Studien sind erforderlich, um die Bedeutung von Chest Pain Units beim Einsatz von Provokationstests aufzuzeigen [51]. Chest Pain Units können dazu dienen, die Aufenthaltsdauer zu reduzieren, die Zahl der Krankenhausaufnahmen und die Kosten zu vermindern sowie die diagnostische Sicherheit und die Lebensqualität zu verbessern [52]. Es gibt allerdings keinen unmittelbaren Beweis dafür, dass Chest Pain Units oder Beobachtungsprotokolle bei Patienten mit einem möglichen ACS zu einer Reduzierung unerwünschter Ereignisse, insbesondere der Sterblichkeit, führen.

\section{Bildgebende Verfahren}

Effektives Screening von Patienten mit ACS-Verdacht, aber negativem EKG und negativen kardialen Biomarkern stellt eine Herausforderung dar. Nichtinvasive Techniken wie CT-Angiographie [53], kardiale Magnetresonanztomographie, myokardiale Perfusionsscintigraphie [54] und Echokardiographie [55] wurden als Möglichkeiten evaluiert, die Niedrigrisikogruppen zu untersuchen und Subgruppen zu identifizieren, die sicher nach Hause entlassen werden können [31, 5658]. Darüber hinaus können Differenzialdiagnosen wie Aortendissektion, Lungen(arterien)embolie, Aortenstenose, hypertrophe Kardiomyopathie, Pleuraerguss oder Pneumothorax erfasst werden. Die Echokardiographie solle deshalb rou- tinemäßig in der Notfallambulanz verfügbar sein und bei allen Patienten mit ACSVerdacht eingesetzt werden. Es sind Studien notwendig, die die Rolle der Echokardiographie in der Prähospitalphase bewerten. Obwohl keine großen multizentrischen Studien vorliegen, gibt es deutliche Hinweise darauf, dass die diagnostischen Möglichkeiten einen frühen und verlässlichen Befund mit Reduzierung der Aufenthaltsdauer und der Kosten ermöglichen, ohne die Zahl kardialer Ereignisse zu erhöhen. Sowohl die Risiken der Strahlenexposition als auch die der iodierten Kontrastmittel sollen bedacht werden, wenn eine kardiale Perfusionsscintigraphie bzw. eine koronare Multidetektor-Computerangiographie (MDTCA) zum Einsatz kommt.

Die MDTCA wurde kürzlich für die Behandlung von Patienten mit akutem Brustschmerz in der Notfallambulanz vorgeschlagen. Sie ist zuverlässig im Vergleich zur invasiven Koronarangiographie, erlaubt differenzialdiagnostische Klärungen und ist sowohl brauchbar als auch praktikabel in der Notfallambulanz [53, 59-63]. Die MDTCA verfügt über ein großes Potenzial, höhergradige Koronarstenosen auszuschließen [64, 65]. Der frühzeitige Einsatz der MDTCA bei Patienten, die sich mit Brustschmerz und einem niedrigen bis mittleren ACS-Risiko in der Notfallambulanz vorstellen, identifiziert rasch ein Gruppe mit besonders niedrigem Risiko (Risiko für ein unerwünschtes kardiales Ereignis $<1 \%$ in 30 Tagen) und erlaubt ein sichere und beschleunigte Entlassung. Durch Vermeidung nicht notwendiger Krankenhausaufnahmen bzw. verlängerter Aufenthaltszeiten hat sich die kardiale MDTCA als effizient erwiesen [66-68]. Bei einer signifikanten Anzahl von ACS-Patienten mit niedrigem Risiko deckt die MDTCA schwere Koronarläsionen auf und ermöglicht so gezielte weitere Diagnostik und therapeutische Intervention. In einer neuen Metaanalyse zeigte die MDTCA eine hohe Sensitivität und eine niedrige Versagenswahrscheinlichkeit von 0,06 und war in der Lage, ein ACS bei Patienten mit niedrigem bis mittlerem Risiko, die sich in der Notfallambulanz mit Brustschmerz vorstellten, auszuschließen [69]. Die Unmöglichkeit aber, mit anatomischen Be- 


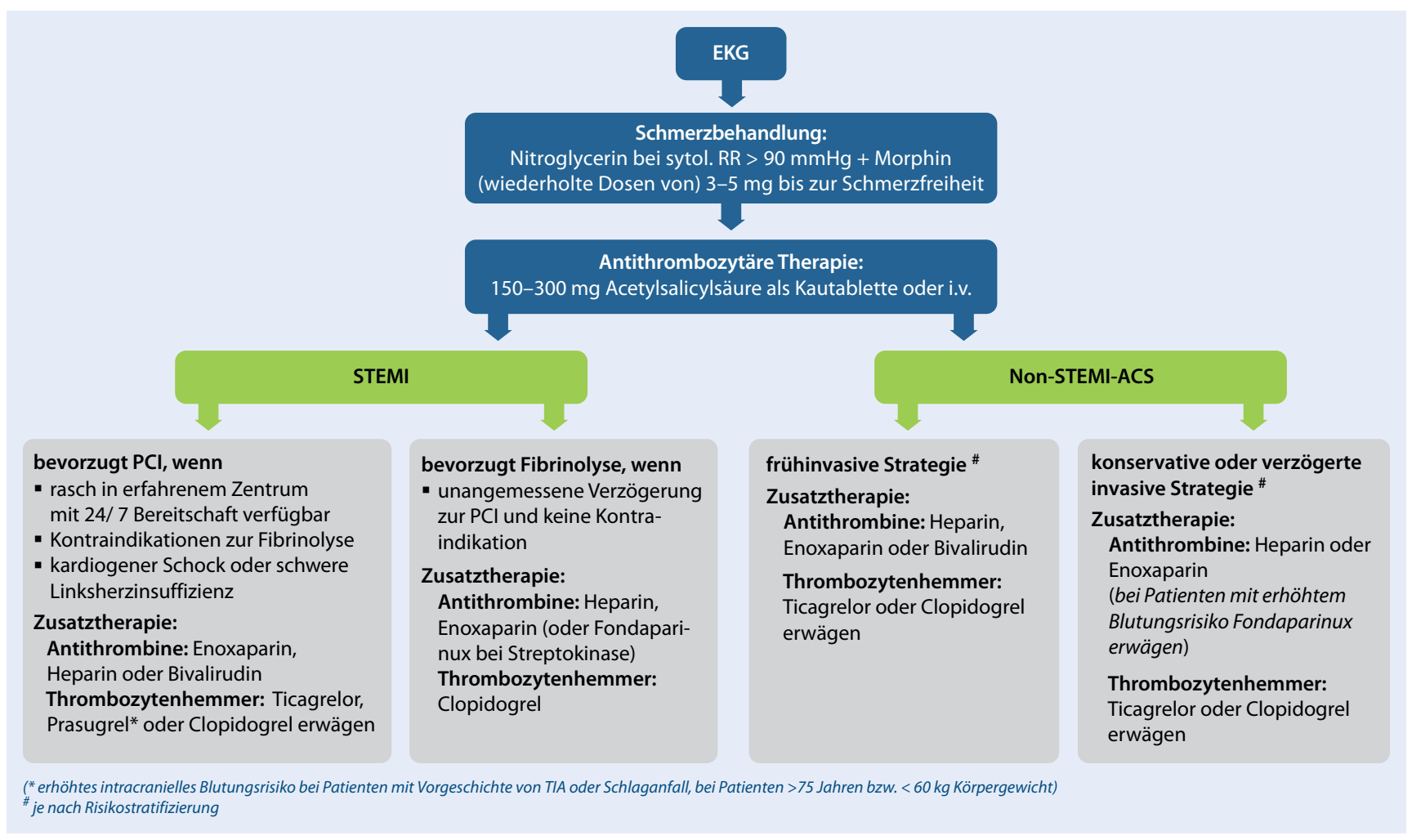

Abb. 2 \ Behandlungspfade bei akutem Koronarsyndrom. EKG Elektrokardiogramm, STEMI myokardialer ST-StreckenHebungs-Infarkt, non-STEMI Myokardinfarkt ohne ST-Strecken-Hebung, $P C I$ perkutane Koronarintervention

funden das Vorhandensein von Ischämien nachzuweisen, das Krebsrisiko durch die Strahlenexposition und die mögliche übermäßige Nutzung lassen noch immer Bedenken gegenüber der Bedeutung dieser Strategie bestehen.

\section{Die symptomatische Behandlung ACS}

\section{Nitrate}

Glyceroltrinitrat (Nitroglycerin) ist eine effektive Therapie gegen ischämischen Brustschmerz und hat günstige hämodynamische Effekte, wie z. B. eine Dilatation venöser Kapazitätsgefäße, eine Dilatation der Koronararterien und, in einem geringeren Ausmaß, der peripheren Arterien. Die Gabe von Nitroglycerin kommt in Betracht, sofern der systolische Blutdruck (SBP) über $90 \mathrm{mmHg}$ liegt und der Patient unter ischämischem Brustschmerz leidet (• Abb. 2). Nitroglycerin kann auch bei der Behandlung einer akuten Lungenstauung hilfreich sein. Nitrate sollen nicht bei Patienten mit Hypotension („systolic blood pressure“, SBP $<90 \mathrm{mmHg}$ ) gege- ben werden, besonders nicht bei gleichzeitiger Bradykardie, und nicht bei Patienten mit Hinterwandinfarkt und Verdacht auf rechtsventrikuläre Beteiligung. Der Einsatz von Nitraten unter diesen Bedingungen kann den Blutdruck und die ventrikuläre Auswurfleistung senken. Nitrate sollen bei kürzlicher Einnahme von 5'-Phosphodiesterasehemmern $(<48 \mathrm{~h})$ nicht eingesetzt werden $[5,9,70,71]$.

Nitroglycerin wird in bis zu drei Einzeldosen zu $0,4 \mathrm{mg}$ alle $5 \mathrm{~min}$ gegeben, sofern der Blutdruck dies erlaubt. Die i.v.-Gabe wird mit einer Dosis von $10 \mu \mathrm{g} /$ min bei persistierendem Schmerz und bei Lungenödem begonnen und zum gewünschten Blutdruckeffekt hochtitriert.

\section{Analgesie}

Morphin ist das Analgetikum der Wahl bei nitrorefraktärem Schmerz und hat auch beruhigende Wirkung, sodass Sedativa in den meisten Fällen unnötig sind. Da Morphin ein Dilatator venöser Kapazitätsgefäße ist, kann es außerdem Vorteile für Patienten mit Lungenstauung haben. Morphin solle in Initialdosen von
3-5 mg i.v. und wiederholt im Abstand von wenigen Minuten gegeben werden, bis der Patient schmerzfrei ist. Vorsicht ist geboten bei eingetrübten Patienten, Hypotension, Bradykardie bzw. bekannter Überempfindlichkeit $[5,9,71]$. Nichtsteroidale entzündungshemmende Medikamente (NSAIDs) sollen bei der Analgesie wegen ihres prothrombotischen Effekts vermieden werden [72].

\section{Sauerstoff}

Es häufen sich Hinweise auf die fragwürdige Rolle der Sauerstoffgabe bei Kreislaufstillstand und ACS. Patienten mit akutem Brustschmerz bei vermutetem ACS benötigen keine zusätzliche Sauerstoffgabe, sofern sie nicht Zeichen von Hypoxie, Atemnot oder Herzversagen aufweisen. Es gibt zunehmend Hinweise darauf, dass eine Hyperoxie schädlich für den Patienten mit unkompliziertem Infarkt sein könnte [73-76].

Bei einem Kreislaufstillstand als Komplikation des ACS entwickelt sich eine Hypoxie schnell. Der ischämische Hirnschaden ist ein wesentlicher Faktor be- 
züglich des neurologisch intakten Überlebens. Eine adäquate Sauerstoffversorgung ist daher essenziell. Nach Wiederherstellung eines spontanen Kreislaufs (ROSC) sollen sowohl Hyperoxie als auch Hypoxie vermieden werden (s. auch Kapitel „Behandlung nach Reanimation") [77]. $100 \%$ Sauerstoff soll gegeben werden, bis die arterielle Sauerstoffsättigung zuverlässig gemessen werden kann. Sobald dies möglich ist, soll die inspiratorische Sauerstoffkonzentration so eingestellt werden, dass eine arterielle Sauerstoffsättigung in der Größenordnung von 94-98\% (bei chronisch obstruktiver Lungenerkrankung von 88$92 \%)$ erreicht wird $[5,71]$.

\section{Kausale Behandlung des akuten ACS}

\section{Thrombozytenaggre- gationshemmer}

Die auf eine Plaqueruptur folgende Thrombozytenaktivierung und -aggregation sind zentrale Mechanismen des ACS. Die antithrombozytäre Therapie ist demzufolge eine grundlegende Behandlungsform des ACS, mit oder ohne ST-Segment-Hebung, mit oder ohne Reperfusions- bzw. Revaskularisierungsbehandlung.

\section{Acetylsalicylsäure (ASA)}

In großen randomisierten kontrollierten Studien wurde eine geringere Sterblichkeit bei ACS, unabhängig von der Reperfusions- bzw. Revaskularisationsstrategie, nachgewiesen, wenn ASA (75-325 mg) hospitalisierten Patienten mit ACS gegeben wurde. Einige Studien haben eine reduzierte Sterblichkeit festgestellt, wenn ASA noch früher verabreicht wurde [7880]. Deshalb soll eine orale Aufsättigungsdosis (150-300 mg einer nicht magensaftresistenten Zubereitung) oder ein i.v.-Präparat so früh wie möglich allen Patienten mit Verdacht auf ACS gegeben werden, sofern keine echte ASA-Allergie oder eine aktive Blutung vorliegt. ASA kann entsprechend örtlicher Regulierungen von der ersten medizinischen Kontaktperson, einem sonstigen Anwesenden oder auf Anweisung eines Leitstellenmitarbeiters gegeben werden.

\section{ADP-Rezeptor-Antagonisten}

Die Inhibierung des ADP-Rezeptors der Thrombozyten durch die Thienopyridine Clopidogrel und Prasugrel (irreversible Blockierung) bzw. durch das CyclopentylTriazolo-Pyrimidin Ticagrelor (reversible Blockierung) führt über die von ASA hervorgerufene Aggregationshemmung hinaus zu einer weiteren Hemmung der Plättchenaggregation. Im Gegensatz zu Clopidogrel sind die Effekte von Prasugrel und Ticagrelor weitgehend unabhängig von einer genetisch bedingten Variabilität des Arzneimittelstoffwechsels bzw. der Arzneimittelaktivierung. Prasugrel und Ticagrelor (reversible Hemmung) führen daher zu einer zuverlässigeren, schnelleren und stärkeren Hemmung der Plättchenaggregation.

Eine große randomisierte Studie, in der eine Aufsättigungsdosis von $300 \mathrm{mg}$ Clopidogrel, gefolgt von $75 \mathrm{mg}$ täglich, mit Prasugrel (Initialdosis $60 \mathrm{mg}$, gefolgt von $10 \mathrm{mg}$ täglich) bei Patienten mit ACS (s. auch Bemerkungen zum non-STEMIACS weiter unten) und geplanter PCI verglichen wurde, resultierte in weniger unerwünschten wesentlichen kardialen Ereignissen (MACE) mit Prasugrel, jedoch war die Blutungsrate höher. Das Blutungsrisiko war deutlich erhöht bei Patienten mit einem Körpergewicht unter $60 \mathrm{~kg}$ und einem Alter von über 75 Jahren [81]. Mit Prasugrel wurde eine signifikant erhöhte Rate intrakranieller Blutungen bei $\mathrm{Pa}$ tienten mit transitorischer ischämischer Attacke (TIA) und/oder Schlaganfall in der Vorgeschichte beobachtet. In einer weiteren Studie bei ACS erwies sich Ticagrelor (Initialdosis $180 \mathrm{mg}$, gefolgt von 2-mal täglich $90 \mathrm{mg}$ ) im Vergleich zu Clopidogrel (Initialdosis 300-600 mg, gefolgt von $75 \mathrm{mg}$ täglich) hinsichtlich Sterblichkeit und Auftreten von MACE als überlegen, ging aber ebenfalls mit einer erhöhten Blutungsrate einher [82].

\section{ADP-Rezeptor-Inhibitoren beim non- STEMI-ACS}

Clopidogrel. Wenn Clopidogrel zusammen mit Heparin und ASA bei Patienten mit Hochrisiko non-STEMI-ACS gegeben wird, verbessert dies das Behandlungsergebnis [83]. Im Falle eines geplanten konservativen Vorgehens soll eine In- itialdosis von $300 \mathrm{mg}$ gegeben werden, bei geplanter PCI-Strategie bevorzugt man eine Dosis von $600 \mathrm{mg}$. Es existiert keine große ACS-Studie, die eine Vorbehandlung mit Clopidogrel, weder 300 noch $600 \mathrm{mg}$, mit einer periinterventionellen Gabe vergleicht.

Prasugrel. Prasugrel (60 mg Initialdosis) kann bei Patienten mit Hochrisiko-nonSTEMI-ACS und geplanter PCI erst gegeben werden, wenn vorab durch Angiographie für die PCI geeignete Stenosen nachgewiesen sind. Die Kontraindikationen (Vorgeschichte mit TIA/Schlaganfall) und das Verhältnis Blutungsrisiko versus Nutzen (Gewicht $<60 \mathrm{~kg}$, Alter $>75$ Jahre) soll beachtet werden. Eine randomisierte kontrollierte Untersuchung, die die Gabe von Prasugrel vor bzw. nach Angiographie verglich, zeigte, dass die Vorbehandlung mit einem höheren Blutungsrisiko verbunden war, ohne die Zahl thrombotischer Ereignisse zu reduzieren. Damit ist Prasugrel von jedweder Vorbehandlungsstrategie bei non-STEMI-ACS inoder außerhalb des Krankenhauses ausgeschlossen, bevor die Koronaranatomie bekannt ist [84].

Ticagrelor. Entsprechend den ESC Guidelines [6] solle Ticagrelor (180 mg Initialdosis) allen Patienten mit non-STEMI-ACS und mittlerem bis hohem Risiko zusätzlich zu ASA gegeben werden, unabhängig davon, ob eine invasive Strategie geplant ist oder nicht. Patienten mit non-STEMI-ACS, die konservativ behandelt werden sollen, sollen Ticagrelor bzw. Clopidogrel erhalten, sobald die Diagnose feststeht. Für eine Empfehlung für oder gegen eine Vorbehandlung mit diesen Substanzen bei geplanter PCI liegen keine ausreichenden Daten vor.

ADP-Rezeptor-Antagonisten bei STEMI

Clopidogrel. Die prähospitale im Vergleich zur intrahospitalen Gabe von Clopidogrel bei STEMI wurde in zwei kleineren Studien untersucht, die die Sicherheit, nicht jedoch wesentliche klinische Nutzeffekte belegten [85, 86]. Dennoch zeigte eine Metaanalyse des Vergleichs der Clopidogrel-Gabe vor bzw. nach PCI (nicht 
prähospital vs. intrahospital!) bei einer STEMI-Patienten-Subgruppe einen signifikanten Vorteil zugunsten der Vorbehandlung hinsichtlich Sterblichkeit und Infarktraten ohne erhöhtes Blutungsrisiko [87]. Obwohl es keine große Studie zum Prä-PCI-Einsatz von Clopidogrel bei Patienten mit STEMI und geplanter PCI gibt, scheint es wahrscheinlich, dass diese Strategie vorteilhaft ist. Da die Thrombozytenaggregationshemmung mit einer höheren Dosis ausgeprägter ist, kann eine so früh wie möglich gegebene Initialdosis von $600 \mathrm{mg}$ bei Patienten mit STEMI und geplanter PCI in Betracht gezogen werden.

Zwei große randomisierte Studien haben Clopidogrel bei Patienten mit STEMI untersucht, die konservativ oder mit Fibrinolyse behandelt wurden $[88,90]$. Eine Studie umfasste Patienten bis zum Alter von 75 Jahren, die mit Fibrinolyse, ASA, einem Antithrombin und einer Initialdosis von $300 \mathrm{mg}$ Clopidogrel [87] behandelt wurden. Die Clopidogrel-Behandlung ergab weniger verschlossene, für den Infarkt verantwortliche Koronargefäße bei Angiographie und weniger Reinfarkte ohne erhöhtes Blutungsrisiko. Die andere Studie untersuchte STEMI-Patienten ohne Altersbegrenzung, die konservativ oder mit Fibrinolyse behandelt werden sollen. In dieser Untersuchung führte Clopidogrel (75 mg täglich ohne Aufsättigungsdosis) im Vergleich zu Placebo zu weniger Todesfällen und einer Reduzierung des kombinierten Endpunkts aus Schlaganfall und Tod [89]. Patienten mit STEMI, die mit Fibrinolyse behandelt werden, sollen deshalb zusätzlich zu ASA und einem Antithrombin Clopidogrel erhalten (300 mg Initialdosis bei einem Alter von bis zu 75 Jahren und $75 \mathrm{mg}$ ohne Aufsättigungsdosis bei Patienten im Alter von über 75 Jahren).

Prasugrel. Prasugrel in einer Aufsättigungsdosis von $60 \mathrm{mg}$ kann bis $24 \mathrm{~h}$ vor, während und auch nach PCI zusätzlich zu ASA und einem Antithrombin bei Patienten mit STEMI und geplanter PCI gegeben werden [91]. Die Kontraindikationen (Vorgeschichte mit TIA/Schlaganfall) und das Verhältnis des Nutzens gegenüber dem Blutungsrisiko bei Patienten mit einem Körpergewicht von $<60$ kg oder einem Alter $>75$ Jahre ist zu berücksichtigen. Es liegen keine Daten zum prähospitalen Einsatz von Prasugrel bzw. zum Einsatz von Prasugrel im Zusammenhang mit Fibrinolyse vor.

Ticagrelor. Ticagrelor kann mit einer Aufsättigungsdosis von $180 \mathrm{mg}$ bei Patienten mit STEMI und geplanter PCI eingesetzt werden. Der Vorteil einer prähospitalen Behandlung im Vergleich zur Gabe einer Initialdosis von $180 \mathrm{mg}$ im Herzkatheterlabor wurde in einer randomisierten kontrollierten Studie an 1862 STEMI-Patienten mit geplanter PCI und einer Symptomdauer von bis zu $6 \mathrm{~h}$ geprüft. Die Studie ergab keinen Nutzen beim Koronarfluss oder bei der Rückbildung der STSegment-Hebung (primärer Endpunkt) bzw. wesentlichen klinischen Endpunkten. Sie zeigte allerdings, dass die prähospitale Gabe von Ticagrelor zu einer reduzierte Rate von definitiven Stentthrombosen führte (OR 0,19; CI 0,04-0,86), ohne gesteigertes Blutungsrisiko [92]. Dieser Endpunkt war jedoch nicht vorab festgelegt und soll nur als hypothesengenerierend betrachtet werden. Es gibt keine Daten zu Ticagrelor im Zusammenhang mit der Fibrinolyse.

Der relative Nutzen der routinemäßigen prähospitalen Gabe eines ADP-Antagonisten bei STEMI-Patienten mit geplanter PCI ist möglicherweise nur marginal und könnte von zusätzlichen Schädigungen übertroffen werden, die im Rahmen größerer Outcome-orientierter, randomisierter kontrollierter Untersuchungen geklärt werden sollen. Im Falle eines Verlegungstransports zur PPCI können ADPAntagonisten nach sorgfältiger Patientenindividueller Abwägung des Nutzen-Risiko-Verhältnisses erwogen werden.

\section{Glykoprotein(Gp)-IIB/ IIIA-Rezeptorblocker}

Der Glykoprotein(Gp)-IIB/IIIA-Rezeptor ist das gemeinsame Endglied der Thombozytenaggregation. Eptifibatid und Tirofiban führen zu einer reversiblen Inhibition, während Abciximab zu einer irreversiblen Inhibition des Gp-IIB/IIIA-Rezeptors führt. Ergebnisse älterer Studien aus der Prästent-Ära unterstützen zumeist den Einsatz dieser Substanzklasse [93]. Neuere Untersuchungen zeigen eher neutrale bis negative Ergebnisse [94] mit Ausnahme der kürzlich veröffentlichten ON-TIME-2-Studie, in der der systematische prähospitale Einsatz von Tirofiban mit dem unterstützenden Einsatz bei der PPCI verglichen wurde. Diese Studie zeigte einen Nutzen der Vorbehandlung mit Gp-IIB/IIIA-Rezeptorblockern hinsichtlich des primären Endpunkts persistierender ST-Strecken-Hebungen und hinsichtlich Noteingriffen bei thrombotischen Komplikationen ohne erhöhtes Blutungsrisiko [95]. Ebenso zeigte eine kürzlich publizierte Metaanalyse von 7 randomisierten Studien an insgesamt 722 Patienten, in der die frühzeitige mit späterer Abciximab-Therapie bei STEMI-Patienten mit geplanter PPCI verglichen wurde, den Vorteil einer frühen Behandlung bezüglich der Offenheit von Koronargefäßen und daraus folgend niedriger Sterblichkeit [96]. Jedoch zeigten fast alle Studien, unabhängig davon, ob sie den Einsatz von Gp-IIB/IIIA-Rezeptorblockern unterstützten, neutral waren oder von negativen Resultaten ausgingen, dass mit ihnen mehr Patienten Blutungen aufwiesen. Insofern liegen zu wenige Daten vor, die eine routinemäßige Vorbehandlung mit Gp-IIB/IIIA-Rezeptorblockergabe bei Patienten mit STEMI oder non-STEMI-ACS unterstützen. Es wird deshalb nicht empfohlen, Gp-IIB/IIIA-Rezeptorblocker zu geben, bevor die Koronaranatomie bekannt ist. Für Hochrisikopatienten mit non-STEMI-ACS kann die Vorbehandlung im Krankenhaus mit Trofiban oder Eptifibatide akzeptabel sein, wohingegen Abciximab nur im Zusammenhang mit der PCI einen Platz hat. Betrachtet man das erhöhte Blutungsrisiko bei Einsatz von Gp-IIb/IIIA-Rezeptorblockern bei zusätzlicher Gabe von Heparinen, sollen alternative Behandlungsstrategien mit Gabe von ADP-Antagonisten in Betracht gezogen werden [97].

\section{Antithrombine}

Unfraktioniertes Heparin (UFH) ist ein indirekter Thrombininhibitor, der in Kombination mit ASA als Zusatz zur Fibrinolyse oder bei der PPCI eingesetzt wird. Es ist ein wichtiger Bestandteil der Behandlung der instabilen Angina pectoris und des STEMI. Die Probleme von 
UFH liegen in dem für individuelle $\mathrm{Pa}$ tienten nicht voraussagbaren antikoagulatorischen Effekt und der Notwendigkeit, es intravenös zu geben und die aktivierte partielle Thromboplastinzeit (aPTT) zu überwachen. Darüber hinaus kann UFH eine Thrombozytopenie verursachen. Seit Erstellung der ERC-Leitlinie 2010 zum ACS wurden mehrere randomisierte Studien durchgeführt, in denen alternative Antithrombine im Vergleich zu UFH zur Behandlung des ACS getestet wurden [98-100]. Diese Alternativen sind charakterisiert durch eine spezifischere Faktor- $\mathrm{X}_{\mathrm{a}}$-Aktivität (niedermolekulare Heparine, LMWH, Fondaparinux) oder sind direkte Thrombininhibitoren (Bivalirudin). Mit den letztgenannten Antithrombinen muss das Koagulationssystem nicht notwendigerweise überwacht werden, und dasThrombozytopenierisiko ist reduziert. Mit Ausnahme von UFH fehlen Studien, die isoliert die prähospitale mit der intrahospitalen Gabe des gleichen Antithrombins vergleichen. Rivaroxaban, Apixaban und andere direkte Thrombinantagonisten haben ihre Indikation bei bestimmten Patientengruppen nach Stabilisation, aber nicht bei der Initialbehandlung des ACS [101].

\section{Antithrombine bei non-STEMI-ACS}

Eine parenterale Antikoagulation wird zum Zeitpunkt der Diagnose unter Berücksichtigung sowohl der Ischämie- als auch der Blutungsrisiken empfohlen, da sie - zusammen mit Thrombozytenaggregationshemmern - das Risiko wesentlicher kardiovaskulärer Ereignisse (MACE) bei Patienten mit non-STEMI-ACS reduziert. Wenn auch die Grundidee der frühen Antithrombin-Therapie in der Vermeidung von MACE liegt, gibt es dennoch keinen eindeutigen Beweis für die Überlegenheit des prähospitalen im Vergleich zum intrahospitalen Beginn der Antithrombin-Therapie.

Verglichen mit UFH (70-100 IU/kg i.v.) verringert Enoxaparin (30 mg i.v., gefolgt von $1 \mathrm{mg} / \mathrm{kg}$ s. c. alle $12 \mathrm{~h}$ ) den kombinierten Endpunkt aus Mortalität, Myokardinfarkt und der Notwendigkeit dringlicher Revaskularisation, wenn es in den ersten 24-36 h nach Symptombeginn eines non-STEMI-ACS eingesetzt wird
[102, 103]. ACT-gesteuerte (ACT, ,activated clotting time") i.v.-Nachinjektionen können nach initialer UFH-Behandlung erwogen werden. Obwohl Enoxaparin mehr geringfügige Blutungen als UFH verursacht, ist die Rate ernster Blutungen nicht erhöht.

Blutungen verschlechtern die Prognose von Patienten mit ACS [104]. Fondaparinux (2,5 mg s. c. täglich) und Bivalirudin (initialer Bolus von $0,1 \mathrm{mg} / \mathrm{kg}$ i.v., gefolgt von einer Infusion von $0,25 \mathrm{mg} / \mathrm{kg}$ ) führen zu weniger Blutungen als UFH [105-107]. Fondaparinux wird wegen des besten Wirksamkeits-Sicherheits-Profils empfohlen, unabhängig von der Behandlungsstrategie. Da Katheterthromben bei Patienten mit PCI beobachtet wurden, ist zusätzliches UFH während einer PCI notwendig [105].

Enoxaparin oder UFH werden für den Fall empfohlen, dass Fondaparinux nicht verfügbar ist. In den Studien an Patienten mit non-STEMI-ACS wurden UFH, Fondaparinux, Enoxaparin und Bivalirudin nur nach Krankenhausaufnahme eingesetzt. Es mag daher unzulässig sein, die Daten auf die prähospitale Situation oder die in der Notfallambulanz zu übertragen.

Wegen des Vorteils weniger Blutungen ist Fondaparinux ein vorzuziehendes Antithrombin. Da aber Fondaparinux und Enoxaparin $^{2}$ bei Niereninsuffizienz akkumulieren können, ist eine Dosisanpassung notwendig. Für Patienten mit geplantem invasivem Vorgehen sind Bivalirudin und Enoxaparin sinnvolle Alternativen zum UFH. Das Blutungsrisiko kann jedoch steigen, wenn zwischen Enoxaparin und UFH gewechselt wird [108]. Nach Durchführung der PCI solle die Antikoagulation beendet werden, falls nicht aus anderen Gründen indiziert.

\section{Antithrombine bei STEMI}

\section{Antithrombine für Patienten, die mit} Fibrinolyse behandelt werden sollen

Enoxaparin und UFH. Die Gabe von UFH ist sinnvoll für die präklinische Fibrinolyse-Therapie bei STEMI-Patienten. Einige Studien an Patienten mit Fibrinolyse bei

\footnotetext{
2 In geringerem Umfang bei einer Kreatininc-
} learance unter $30 \mathrm{ml} / \mathrm{h}$ auch Bivalirudin.
STEMI haben jedoch gezeigt, dass die Behandlung mit Enoxaparin statt UFH, unabhängig von dem verwendeten Fibrinolytikum, zu besseren klinischen Resultaten führte, allerdings auch einherging mit einer erhöhten Blutungsrate bei älteren Patienten über 75 Jahre und einem geringen Körpergewicht unter 60 kg [109]. Reduzierte Dosen von Enoxaparin bei älteren Patienten und geringem Körpergewicht führten bei geringerer Blutungsneigung weiterhin zu günstigeren Ergebnissen [110].

Dosierung bei Enoxaparin: Bei Patienten $<75$ Jahre folgt einem initialen Bolus von $30 \mathrm{mg}$ i.v. eine Dosierung von $1 \mathrm{mg} /$ $\mathrm{kg}$ alle $12 \mathrm{~h}$ wobei die erste s. c.-Gabe kurz nach dem i.v.-Bolus gegeben werden soll. Patienten $\geq 75$ Jahre sollen mit $0,75 \mathrm{mg} / \mathrm{kg}$ s. c. alle $12 \mathrm{~h}$ ohne initialen i.v.-Bolus behandelt werden. Patienten mit bekannter eingeschränkter Nierenfunktion (Kreatininclearance $<30 \mathrm{ml} / \mathrm{min}$ ) können mit $1 \mathrm{mg} / \mathrm{kg}$ s. c. einmal täglich behandelt werden oder sollen UFH erhalten. Zur Empfehlung anderer niedermolekularer Heparine fehlen ausreichende Daten.

Fondaparinux. In mehreren Studien wurde ein neutrales Ergebnis bzw. eine Überlegenheit von Fondaparinux im Vergleich zu UFH bei Fibrinolyse von STEMI-Patienten gezeigt [105]. Fondaparinux (initial 2,5 mg s. c., gefolgt von täglich 2,5 mg s. c.) kommt insbesondere beim Einsatz von nichtfibrinspezifischen Thrombolytika (Streptokinase) bei Patienten mit einer Plasma-Kreatinin-Konzentration $<3$ mg/l $(<250 \mathrm{Mikromol} / \mathrm{l})$ in Betracht. Für den Fall einer geplanten PPCI solle Enoxaparin oder UFH bevorzugt werden.

Bivalirudin. Es liegen zu wenige Daten vor, um Bivalirudin anstelle von UFH bzw. Enoxaparin für die Fibrinolyse-Therapie bei STEMI-Patienten zu empfehlen. Da der Wechsel von Antithrombinen das Blutungsrisiko erhöhen kann, soll das initiale Medikament beibehalten werden, mit der Ausnahme von Fondaparinux, wo UFH zusätzlich notwendig wird, wenn eine ergänzende PCI geplant ist [108].

Antithrombine bei STEMI-Patienten, die mit primärer $\mathrm{PCI}$ (PPCI) behandelt werden sollen. Nach Veröffentlichung 
der ERC-Leitlinien 2010 wurden Studien durchgeführt, in denen verschiedene Antithrombine prähospital bei Patienten mit STEMI und geplanter PPCI eingesetzt wurden [98, 99, 111]. Mit Ausnahme von UFH [112] jedoch besteht ein Mangel an Studien zum Vergleich der prähospitalen mit der intrahospitalen Gabe desselben Antithrombins, d. h. der Bedeutung eines früheren Therapiebeginns. Die Therapieempfehlungen für diese Bedingungen müssen daher vorwiegend aus intrahospitalen Studien extrapoliert werden, ohne dass bis zum Vorliegen spezifischerer Studienergebnisse ein Vorteil prähospitalen Therapiebeginns sicher bewiesen ist.

UFH. Die prähospitale intravenöse Injektion von $500 \mathrm{mg}$ ASA und > 5000 IU UFH führte in einer Beobachtungsstudie zu einer höheren Rate an TIMI-Fluss 2 und 3 sowie TIMI-Fluss Grad 3 bei Erstangiographie im Vergleich zur späteren Gabe [112]. Es ergab sich jedoch kein Einfluss auf die Infarktgröße und die 30-Tage-Sterblichkeit.

Enoxaparin. In einer größeren randomisierten Studie wurde Enoxaparin mit UFH bei geplanter PPCI bei STEMI verglichen. Bei $71 \%$ der Patienten wurde die Antithrombingabe im Rettungswagen begonnen [99]. Die Studie ergab keinen Unterschied in Bezug auf primären Endpunkt aus Tod, misslungener Intervention oder wesentlicher Blutung, aber geringere Raten verschiedener kombinierter sekundärer Endpunkte wie z. B. Tod, erneutes ACS und dringende Revaskularisierung. Verschiedene Register und kleinere Studien zeigten ebenfalls ein günstiges oder neutrales Ergebnis, wenn Enoxaparin mit UFH bei PPCI (mit großzügigem Einsatz von ADP-Antagonisten bzw. Gp-IIB/IIIA-Rezeptorblockern) [113] verglichen wurde. Enoxaparin ist daher als eine effektive und sichere Alternative zu UFH anzusehen und solle eventuell dem UFH auch unter prähospitalen Bedingungen vorgezogen werden. Die Datenlage ist unzureichend, um andere niedermolekulare Heparine als Enoxaparin für die PPCI bei STEMI zu empfehlen. Der Wechsel von UFH zu Enoxaparin oder umgekehrt kann $\mathrm{zu}$ einem erhöhten Blutungsrisiko führen und soll deshalb vermieden werden [108]. Eine Dosisanpassung ist für Enoxaparin bei Niereninsuffizienz notwendig.
Bivalirudin. In zwei großen randomisierten Studien wurde nachgewiesen, dass beim Vergleich von Bivalirudin mit UFH plus Gp-IIB/IIIA-Rezeptorblockern bei Patienten mit STEMI und geplanter PPCI unter Bivalirudin weniger Blutungen auftraten und die Langzeitsterblichkeit sank $[114,115]$. Mehrere andere Studien und Fallserien zeigten ebenfalls bessere oder neutrale Ergebnisse für Bivalirudin im Vergleich mit UFH. Die Machbarkeit und Sicherheit der prähospitalen Anwendung wurde ebenso gezeigt wie geringere Blutungsraten, wenn Bivalirudin mit historischen Kontrollgruppen verglichen wurde $[100,111]$. In neueren Untersuchungen mit weniger intensivem Einsatz von Gp-IIB/ IIIA-Rezeptorblockern bzw. einer modifizierten Plättchenhemmerstrategie waren die Unterschiede bei größeren Blutungen nur noch gering, während die Ergebnisse bei den ischämischen Endpunkten mit Bivalirudin neutral oder sogar schlechter waren $[116,117]$. In einer weiteren Studie wurde die prähospitale Einleitung einer Bivalirudin-Therapie mit UFH und optionalem Einsatz von Gp-IIB/IIIA-Rezeptorblockern verglichen (gegeben bei $69 \%$ der Patienten). Blutungen, nicht jedoch Todesfälle waren mit Bivalirudin seltener. Die Zahl der Stent-Thrombosen war mit Bivalirudin wie in anderen Studien in den ersten 24 h nach PCI höher $[98,118]$. Wenn die reduzierte Blutungshäufigkeit gegenüber der höheren Rate von StentThrombosen abgewogen wird, kann Bivalirudin unverändert als eine Alternative zu UFH bei der PPCI des STEMI angesehen werden.

Fondaparinux. Beim Vergleich mit UFH ergab der Einsatz von Fondaparinux ähnliche klinische Ergebnisse wie UFH im Zusammenhang mit der PPCI [105]. Jedoch erforderte die Thrombusbildung an Kathetern zusätzliche Gabe von UFH. Fondaparinux kann daher nicht bei STEMI und geplanter PPCI empfohlen werden.

\section{Reperfusionsstrategie bei Patienten mit STEMI}

Die Reperfusionstherapie bei Patienten mit STEMI ist der wichtigste Fortschritt in der Behandlung des Myokardinfarkts in den letzten 30 Jahren. Bei Patienten mit STEMI und einer Symptomdauer von bis zu 12 h solle eine Reperfusionsbehandlung mit der am besten geeigneten verfügbaren Strategie so schnell wie möglich eingeleitet werden [119-122]. Die Reperfusion kann mit einer Fibrinolyse, der PPCI oder einer Kombination beider Verfahren erreicht werden. Die Wirksamkeit der Reperfusionstherapie ist entscheidend abhängig vom Zeitintervall vom Symptombeginn bis zum Beginn der Reperfusion. Die Fibrinolyse ist besonders in den ersten 2 bis $3 \mathrm{~h}$ nach Symptombeginn wirksam, die Wirksamkeit der PPCI ist nicht so stark zeitabhängig.

\section{Fibrinolyse}

Eine Metaanalyse von drei randomisierten kontrollierten Studien (RCTs), die 531 Patienten umfasste, ergab einen Vorteil der prähospitalen gegenüber der intrahospitalen Thrombolyse beim Überleben bis zur Krankenhausentlassung ohne zusätzliche Schädigung im Sinne wesentlicher oder intrakranieller Blutungen [123125]. Ein wirksames und sicheres System für die prähospitale Lysetherapie erfordert angemessene Möglichkeiten für die Diagnose und Therapie des Myokardinfarkts und seiner Komplikationen. Die Fibrinolyse kann sicher eingesetzt werden von Paramedics (Rettungsassistenten), Krankenschwestern oder Ärzten, die einem bewährten Protokoll folgen, sowie ein umfassendes Trainings- und Qualitätssicherungsprogramm unter medizinischer Aufsicht durchlaufen haben [126]. Idealerweise sollen sie in der Lage sein, mit erfahrenen Krankenhausärzten (z. B. Notärzten, Kardiologen) zu kommunizieren. Der entscheidende Vorteil der prähospitalen Fibrinolyse wird bei signifikanten Transportzeiten von > 30-60 min erreicht. Die RCTs mit Vorteil für die prähospitale Fibrinolyse wurden unter Systembedingungen durchgeführt, die eine mittlere Zeitdifferenz zwischen prähospitalem und intrahospitalem Therapiebeginn von 33-52 min aufwiesen. Die Transportzeiten lagen im Median bei 38-60 min. Bei abnehmender Transportzeit gehen die Vorteile verloren. Die prähospitale Fibrinolyse bei Patienten mit STEMI oder einem ACS mit vermutlich neuem Linksschen- 
Tab. 1 Kontraindikationen für die Fibrinolyse

\begin{tabular}{|c|c|}
\hline \multirow[t]{6}{*}{ Absolute Kontraindikationen } & $\begin{array}{l}\text { Hämorrhagischer Schlaganfall oder Schlaganfall unklarer Ätiolo- } \\
\text { gie in den letzten } 6 \text { Monaten }\end{array}$ \\
\hline & Zentralnervöse Schädigung oder Neoplasien \\
\hline & $\begin{array}{l}\text { Zustand nach vor kurzem vorausgehendem größeremTrauma, } \\
\text { Operation oder Kopfverletzung (innerhalb der letzten } 3 \text { Wochen) }\end{array}$ \\
\hline & Gastrointestinale Blutung innerhalb des letzten Monats \\
\hline & Bekannte Blutungsneigung \\
\hline & Aortendissektion \\
\hline \multirow[t]{9}{*}{ Relative Kontraindikationen } & TIA in den vorausgehenden 6 Monaten \\
\hline & Orale Antikoagulantientherapie \\
\hline & Schwangerschaft innerhalb 1 Woche nach Entbindung \\
\hline & Nicht komprimierbare Punktionen \\
\hline & Traumatische Wiederbelebung \\
\hline & Therapierefraktäre Hypertonie (systolischer RR $>180$ mmHg) \\
\hline & Fortgeschrittene Lebererkrankung \\
\hline & Bakterielle Endokarditis \\
\hline & Aktives peptisches Magengeschwür \\
\hline
\end{tabular}

kelblock (LBBB) ist demnach vorteilhaft. Die Wirksamkeit ist kurz nach Symptombeginn am größten. Patienten mit einem ACS und Zeichen eines STEMI (oder einem vermutlich neuen LBBB oder einem strikt posterioren Infarkt), die primär in der Notfallambulanz gesehen werden, sollen so rasch wie möglich eine Fibrinolyse erhalten, sofern ein schneller $\mathrm{Zu}$ gang zu einer PPCI nicht gegeben ist.

\section{Risiken der fibrinolytischen Therapie}

Medizinalpersonen, die eine fibrinolytische Therapie einsetzen, müssen die Kontraindikationen und Risiken kennen (• Tab. 1). Bei Patienten mit großen Infarkten (erkennbar an ausgedehnten EKG-Veränderungen) ist die Wahrscheinlichkeit eines Nutzens der fibrinolytischen Behandlung am größten. Der Gewinn ist weniger eindrucksvoll bei inferioren im Vergleich zu anterioren Infarkten. Ältere Patienten haben absolut ein höheres Sterberisiko, aber der absolute Nutzen der fibrinolytischen Therapie ähnelt dem bei jüngeren Patienten. Das Risiko intrakranieller Blutungen ist erhöht bei Patienten mit einem systolischen Blutdruck $>180 \mathrm{mmHg}$ und stellt eine relative Kontraindikation dar. Das Risiko intrakranieller Blutungen ist weiterhin abhängig von dem gewählten Fibrinolytikum, dem Antithrombin und der Antiplättchentherapie. Eine Alternative bei
Patienten $>75$ Jahre kann bei Einsatz von Enoxaparin eine Halbierung der Tenecterakranieller Blutungen ohne Effizienzverlust reduziert werden kann $[127,128]$.

\section{Primäre perkutane Intervention (PPCl)}

Die Koronarangioplastie mit oder ohne Stenteinlage ist zur bedeutsamsten Sofortbehandlungsstrategie für Infarktpatienten geworden. Eine PPCI in einem erfahrenen Zentrum mit erster Balloninsufflation kurz nach erstem Patientenkontakt von einem erfahrenen Untersucher durchgeführt, bei dem ein hoher Trainingsstatus besteht, ist die bevorzugte Behandlungsform, da sie die Morbidität und Mortalität im Vergleich zur sofortigen Fibrinolyse senkt [129].

\section{Fibrinolyse vs. primäre $\mathrm{PCI}(\mathrm{PPCl})$}

Der Einsatz der PPCI war bisher limitiert durch die mangelnde Verfügbarkeit von Katheterlabors, ausreichend trainierten Klinikern und der Zeitverzögerung bis zur ersten Balloninsufflation. Die Fibrinolyse ist eine breit verfügbare Reperfusionsstrategie. Beide Behandlungswege sind gut eingeführt und waren Gegenstand großer randomisierter Multizenterstudien in den letzten Jahrzehnten. Die Zeit vom Symptombeginn und die PPCIplase-Dosierung sein, womit die Rate int- bedingte Zeitverzögerung (Intervall von der Diagnose bis zur ersten Balloninsufflation minus dem Zeitintervall Diagnose - Venenpunktion bei Fibrinolyse) sind die Kernfragen bei der Auswahl der am besten geeigneten Reperfusionsstrategie.

Die Fibrinolyse ist am effektivsten bei Patienten, die in den ersten 2 bis $3 \mathrm{~h}$ nach Symptombeginn gesehen werden. Sie erweist sich als günstig im Vergleich zur PPCI, wenn sie innerhalb von $2 \mathrm{~h}$ nach Symptombeginn eingeleitet wird und mit einer „Rettungs-PCI“ (Rescue-PCI; PCI bei vermutetem Fibrinolyse-Versagen) oder einer verzögerten PCI kombiniert wird [40, 130, 131]. In den randomisierten Studien, in denen die PPCI mit der Fibrinolyse-Therapie verglichen wurde, war die typische Verzögerung von der Entscheidung bis zum Beginn der PPCI-Behandlung bzw. der Fibrinolyse weniger als 60 min. In Registern, die den Normalzustand realistischer wiedergeben, variierte die akzeptable PPCI-bedingte Zeitverzögerung (d. h. das Zeitintervall von der Diagnose bis zur ersten Balloninsufflation minus dem Zeitintervall Diagnose - Kanüle bei Fibrinolyse), in der die Überlegenheit der PPCI gegenüber der Fibrinolyse nachzuweisen war, beträchtlich zwischen 45 min und etwa 180 min in Abhängigkeit von den Bedingungen des Patienten (Alter, Infarktlokalisation und Symptomdauer). In STEMI-Registern überschritt die systembedingte Verzögerung zur PCI in $58 \%$ der Fälle 120 min [132]. Ein kontinuierliches Monitoring der Systemleistungsfähigkeit wird daher benötigt, um optimale Abläufe und Ergebnisse für Infarktpatienten zu erzielen.

Bei jüngeren Patienten, die früh mit großen Vorderwandinfarkten gesehen werden, kann eine Verzögerung zur PPCI von 60 min inakzeptabel sein, während bei spät gesehenen Patienten ( $>3$ h seit Beginn der Symptome) PPCI-bedingte Verzögerungen von bis zu 120 min angemessen sein können [133]. Das Vorhandensein gewisser Zusatzerkrankungen, wie eine frühere koronare BypassOperation, Diabetes oder Niereninsuffizienz, sind zusätzliche Faktoren, die bei der Wahl der besten Therapie mit berücksichtigt werden müssen [134]. 
Die Verzögerung zur PPCI kann durch Verbesserung des Versorgungssystems wesentlich verkürzt werden $[135,136]$ :

- Ein prähospitales EKG soll so früh wie möglich registriert und hinsichtlich einer möglichen STEMI-Diagnose bewertet werden. Damit kann die Sterblichkeit, sowohl bei Patienten, die für eine PPCI als auch für solche, die für eine Fibrinolyse vorgesehen sind, reduziert werden.

- Das Erkennen eines STEMI kann dadurch bewerkstelligt werden, dass das EKG ins Krankenhaus übertragen wird, wo Ärzte vor Ort die Interpretation vornehmen oder intensiv trainierte Krankenschwestern oder Rettungsassistenten es mit oder ohne Unterstützung einer Computer-EKGAuswertung beurteilen.

- Wenn die PPCI die geplante Strategie ist, trägt eine prähospitale Alarmierung des Katheterlabors für die PPCI zur Mortalitätssenkung bei [40].

Folgende zusätzliche Elemente tragen zu einem effektiven System bei:

- Die Einsatzbereitschaft des Herzkatheterlabors innerhalb von $20 \mathrm{~min}$ über $24 \mathrm{~h}$ am Tag an 7 Tagen in der Woche.

- Die Rückmeldung der Daten des realen Zeitverlaufs vom Symptombeginn bis zur PCI.

Für Patienten mit einer Kontraindikation für eine Fibrinolyse soll, statt auf eine Reperfusionstherapie gänzlich zu verzichten, eine PCI, unabhängig von der zeitlichen Verzögerung, angestrebt werden. Für Patienten im kardiogenen Schock ist die PCI (oder koronare Bypass-Operation) die zu bevorzugende Therapie. An die Fibrinolyse soll nur im Falle einer wesentlichen Zeitverzögerung gedacht werden.

\section{Auswahl für den Interhospi- taltransport zur primären $\mathrm{PCI}$}

Bei der Mehrzahl der Patienten mit einem ablaufenden STEMI wird die Diagnose in der Prähospitalphase oder in der Notfallambulanz eines Krankenhauses ohne PCI-Möglichkeit gestellt. Demzufolge müssen Entscheidungen hinsichtlich der bestmöglichen Strategie für die Revasku- larisierung gefällt werden. Für die Prähospitalphase gibt es Daten, die zeigen, dass obwohl die prähospitale Fibrinolyse dem sofortigen Transport zur PCI hinsichtlich der Mortalität nicht unterlegen ist, sie mit einem erhöhten Risiko für intrakranielle Blutungen einhergeht. Wenn die PCI innerhalb von 60-90 min durchgeführt werden kann, wird die sofortige Zuweisung des Patienten zur PCI gegenüber der prähospitalen Fibrinolyse bevorzugt [40, 127, 137-139].

Wenn ein Patient mit STEMI zunächst in der Notfallambulanz eines Krankenhauses ohne PCI-Möglichkeit gesehen wird, zeigen Daten aus 8 RCTs [140-147], in denen 3119 Patienten eingeschlossen wurden, dass der sofortige Transfer zur PPCI im Vergleich zur Fibrinolyse vor Ort und Transport ausschließlich zur Rescue-PCI hinsichtlich Sterblichkeit, Reinfarkt und Schlaganfall überlegen ist, ohne dass sich Hinweise für anderweitige Schädigungen ergeben. Deshalb soll bei Patienten, die in einer Notfallambulanz eines Krankenhauses ohne PCI-Möglichkeit gesehen werden, eine notfallmäßige Verlegung in ein PCI-Zentrum bedacht werden, sofern die PPCI innerhalb angemessener Zeitgrenzen erfolgen kann.

Es ist weniger sicher, ob eine sofortige Fibrinolyse (prähospital oder im Krankenhaus) bzw. der Transfer zur PPCI bei jüngeren Patienten mit Vorderwandinfarkt und einer Symptomdauer von 2-4 h günstiger ist [133]. Die Verlegung von STEMI-Patienten, die nach mehr als $3 \mathrm{~h}$ und bis zu $12 \mathrm{~h}$ nach Symptombeginn gesehen werden, ist dagegen sinnvoll, sofern der Transport schnell möglich ist.

\section{Die Kombination von \\ Fibrinolyse und perkutaner Koronarintervention}

Die Fibrinolyse und die PCI können in einer Vielzahl von Kombinationen eingesetzt werden, um den koronaren Blutfluss wiederherzustellen und aufrechtzuerhalten. Es gibt verschiedene Wege, in denen die beiden Verfahren kombiniert werden können. Es mangelt etwas an einer Eindeutigkeit der Nomenklatur, in der die PCI innerhalb dieser Vorgehensweise beschrieben wird. Der Begriff „facilitated PCI“ wird benutzt, um eine PCI unmittelbar nach Fibrinolyse zu beschreiben, der Begriff , pharmakoinvasive Strategie" bezieht sich auf eine PCI, die 3-24 h nach Fibrinolyse vorgenommen wird, und der Begriff Rescue-PCI ist definiert als eine PCI, die wegen Lyseversagen durchgeführt wird, nachgewiesen durch eine STSegment-Resolution von weniger als $50 \%$ 60-90 min nach Abschluss der Fibrinolyse-Therapie. Diese Strategien unterscheiden sich von einer routinemäßigen Angiographie und Intervention, die mehrere Tage nach erfolgreicher Fibrinolyse durchgeführt wird. Eine sofortige Routineangiographie nach Fibrinolyse geht mit einer erhöhten Rate intrakranieller und weiterer wesentlicher Blutungen einher, ohne irgendeinen Vorteil bezüglich Sterblichkeit und Reinfarkten [148-152].

Es ist sinnvoll, eine Angiographie und PCI bei Patienten mit Fibrinolyse-Versagen durchzuführen, erkennbar an klinischen Zeichen und/oder unzureichender ST-Segment-Resolution [153]. Im Falle einer klinisch erfolgreichen Fibrinolyse (erkennbar am klinischen Bild und einer ST-Segment-Resolution von $>50 \%$ ) verbessert eine um einige Stunden nach Fibrinolyse verzögerte Angiographie (der sog. pharmakoinvasive Weg) das Ergebnis. Diese Strategie schließt die frühe Verlegung zur Angiographie und, wenn nötig, PCI nach Fibrinolyse ein. Daten aus 7 RCTs mit 2355 Patienten [138, 146, $154-$ 158] ergaben Vorteile bezüglich weniger Reinfarkte bei früher regelhafter Verlegung zur Angiographie nach 3-6 h (bis zu $24 \mathrm{~h}$ ) in den ersten $24 \mathrm{~h}$ nach Fibrinolyse im Vergleich zur reinen Verlegung auf die Rescue-PCI nach Fibrinolyse im Krankenhaus (OR 0,57; 95\%-CI 0,38-0,85). Es ergaben sich keine Vorteile bezüglich der kurzzeitigen und Einjahressterblichkeit bzw. Hinweise für Nachteile im Sinne wesentlicher oder intrakranieller Blutungen.

Daten von zwei RCTs [138, 159] und einer nicht randomisierten Studie [160] ergaben keinen Nutzen durch den Transfer zur sofortigen PPCI im Vergleich zur Fibrinolyse-Therapie, gefolgt von einem Routinetransfer zur PCI 3-24 h später. Deshalb ist für den Fall, dass eine PPCI nicht kurzfristig durchgeführt werden kann, eine Fibrinolyse vor Ort, gefolgt von einer Angiographie 3-24 h später, eine sinnvolle Alternative. 


\section{Besondere Situationen}

\section{Kardiogener Schock}

Das ACS ist die häufigste Ursache des kardiogenen Schocks, hauptsächlich verursacht durch ein großes Ischämie-Areal bzw. eine mechanische Komplikation des Myokardinfarkts. Er ist nicht häufig. Die Kurzzeitsterblichkeit des kardiogenen Schocks beträgt $40 \%$ [161] im Kontrast zu einer guten Lebensqualität der Patienten, die lebend entlassen werden. Eine frühe invasive Strategie (primäre PCI, PCI früh nach Fibrinolyse) ist bei den Patienten indiziert, die für eine Revaskularisation infrage kommen [162]. Beobachtungsstudien erlauben die Annahme, dass dieses Vorgehen auch für ältere Patienten (über 75 Jahre) von Vorteil sein könnte. Obwohl häufig in der täglichen Praxis verwendet, gibt es keine Belege, die den Einsatz der intraaortalen Ballonpumpe (IABP) unterstützen [161].

Bei Patienten mit inferiorem Infarkt, klinischen Schockzeichen und Lungenauskultation ohne Stauung besteht der Verdacht auf einen rechtsventrikulären Infarkt. Eine ST-Segment-Hebung $\geq 0,1 \mathrm{mV}$ in Ableitung V4R ist ein guter Indikator für einen rechtsventrikulären Infarkt. Diese Patienten haben eine Krankenhaussterblichkeit von bis zu $30 \%$ und erheblichen Nutzen von einer Reperfusionstherapie. Nitrate und andere Vasodilatoren müssen vermieden und die Hypotension mit intravenöser Flüssigkeit behandelt werden.

\section{Reperfusion nach erfolgreicher Wiederbelebung}

Das ACS wird oft von einem akuten Koronarverschluss oder einer hochgradigen Koronarstenose begleitet und ist eine häufige Ursache des prähospitalen Kreislaufstillstands (OHCA). In einer vor kurzem veröffentlichten Metaanalyse lag die Prävalenz akuter Koronarläsionen zwischen $59 \%$ und $71 \%$ bei OHCA-Patienten ohne offensichtliche nicht kardiale Ursache des Ereignisses [163]. Nach Veröffentlichung einer ersten Studie [164] haben viele Observationsstudien gezeigt, dass die notfallmäßige Untersuchung im Herzkatheterlabor einschließlich früher perkutaner Intervention (PCI) bei Patienten mit wieder vorhandenem Spontankreislauf (ROSC) nach Kreislaufstillstand praktikabel ist [165]. Das invasive Vorgehen (d. h. frühe Koronarangiographie, gefolgt von sofortiger PCI, wenn diese notwendig erscheint) bei dieser Patientengruppe, insbesondere bei Patienten mit langwieriger Reanimation und Vorliegen unspezifischer EKGKriterien, ist umstritten, wegen des Fehlens spezifischer Ergebnisse und der erheblichen Beanspruchung von Ressourcen (einschließlich der Verlegung von Patienten in PCI-Zentren).

PCI nach ROSC bei STEMI. Die höchste Inzidenz von akuten Koronarläsionen findet sich bei Patienten mit ST-Strecken-Elevation bzw. Linksschenkelblock (LBBB) in EKGs, die nach ROSC abgeleitet werden. Es gibt keine randomisierte Studie, aber viele Beobachtungsstudien berichteten über einen Vorteil bei invasivem Vorgehen hinsichtlich Überleben und neurologischem Ergebnis. Es ist hoch wahrscheinlich, dass das frühe invasive Vorgehen eine Strategie bei Patienten mit STEMI darstellt, die mit einem bedeutenden klinischen Vorteil einhergeht. In einer kürzlich durchgeführten Metaanalyse wurde gezeigt, dass eine frühe Angiographie mit einer Reduktion der Krankenhaussterblichkeit (OR 0,35; 0,31$0,41)$ und einem besseren neurologischen Resultat (OR 2,54; 2,17-2,99) verbunden ist [40]. Allerdings gehörten die Patienten, die einer frühen Angiographie unterzogen wurden, zu einer hoch selektionierten Gruppe mit einem höheren Anteil männlicher Patienten, Kammerflimmern, Augenzeugen bei Kreislaufstillstand, therapeutischer Hypothermie und intensiverer linksventrikulärer Unterstützung. Diabetes mellitus, Nieren- bzw. Herzinsuffizienz waren weniger häufig bei diesen $\mathrm{Pa}-$ tienten.

Auf dem Hintergrund der vorhandenen Daten soll die notfallmäßige Evaluierung im Herzkatheterlabor (und sofortige PCI, wenn notwendig) bei ausgewählten erwachsenen Patienten mit ROSC nach vermutet kardialbedingtem OHCA mit ST-Segment-Elevation im EKG durchgeführt werden.

Beobachtungsstudien weisen auch darauf hin, dass optimale Ergebnisse nach OHCA mit einer Kombination aus gezieltem Temperaturmanagement und
PCI erreicht werden. Mit einem standardisierten Protokoll zur Nachbehandlung nach Kreislaufstillstand kann dies festgelegt werden, auch als Teil einer Gesamtstrategie zur Verbesserung des neurologisch intakten Überlebens dieser Patientengruppe.

PCI nach ROSC bei Patienten ohne STStrecken-Hebung. Im Gegensatz zu den Zeichen eines ACS bei Patienten ohne Kreislaufstillstand sind die empfohlenen diagnostischen Methoden zum Nachweis einer koronaren Ischämie in dieser Situation weniger verlässlich. Sowohl die Sensitivität als auch die Spezifität klinischer Daten, des EKG und der Biomarker zum Nachweis eines akuten Koronarverschlusses sind in der Diskussion [167]. Insbesondere zeigt sich, dass auch die Abwesenheit von ST-Hebungen mit einem ACS bei Patienten mit ROSC nach OHCA verbunden sein kann [168]. Bei den Patienten ohne ST-Hebung sind die Daten bezüglich eines möglichen Nutzens einer notfallmäßigen Abklärung im Herzkatheterlabor widersprüchlich - sie kommen alle aus Beobachtungsstudien [169, 170] oder Subgruppenanalysen [171]. Es ist sinnvoll, eine notfallmäßige Abklärung im Herzkatheterlabor nach ROSC bei Patienten mit dem höchsten Risiko für eine koronare Ursache des Kreislaufstillstands zu diskutieren. Eine Reihe von Faktoren, wie Alter des Patienten, Dauer der Reanimation, hämodynamische Instabilität, zugrunde liegende Rhythmusstörung, der neurologische Status bei Aufnahme und die Wahrscheinlichkeit einer kardialen Ursache, kann die Entscheidung zur Intervention beeinflussen. In einem kürzlich erschienenen Konsensuspapier der European Association for Percutaneous Cardiovascular Interventions (EAPCI) wurde betont, dass bei Patienten mit OHCA bei Vorhandensein von ST-Hebungen sofort eine Herzkatheteruntersuchung erfolgen soll bzw. bei anderen Patienten ohne eindeutigen Nachweis einer nicht koronaren Ursache des Kreislaufstillstands diese sobald wie möglich (in weniger als $2 \mathrm{~h}$ ) in Betracht gezogen werden muss, insbesondere wenn sie hämodynamisch instabil sind [172]. Bei Patienten, die nicht in einem PCI-Zentrum erstbehandelt werden, solle die Verlegung zur Angiographie und, 
wenn indiziert, PPCI auf der Grundlage einer individuellen Abwägung zwischen dem erwarteten Nutzen einer frühen Angiographie und den Risiken des Patiententransports erfolgen.

\section{Präventive Maßnahmen}

Präventive Maßnahmen bei Patienten mit ACS sollen früh nach Krankenhausaufnahme begonnen und laufende Therapien fortgesetzt werden. Die Präventivbehandlung verbessert die Prognose durch Reduzierung unerwünschter gravierender kardiovaskulärer Ereignisse. Die medikamentöse Prävention umfasst Betablocker, Inhibitoren des , angiotensin converting enzyme" (ACE-Inhibitoren)/Angiotensin-Rezeptorblocker (ARB) und Statine ebenso wie die Basisbehandlung mit ASA und, sofern indiziert, ADP-Antagonisten.

\section{Betablocker}

Verschiedene Studien, hauptsächlich aus der Vorreperfusionszeit zeigen eine reduzierte Mortalität, Häufigkeit von Reinfarkten und Myokardruptur wie auch seltener Kammerflimmern und supraventrikuläre Arrhythmien bei Patienten, die mit einem Betablocker therapiert wurden. Die Betablocker-Studien unterscheiden sich stark bezüglich des Zeitpunkts des Therapiebeginns. Es mangelt an Daten aus der Präklinik oder Notaufnahme. Außerdem deuten die Studien auf ein höheres Risiko für einen kardiogenen Schock durch i.v.Gabe von Betablockern bei STEMI-Patienten hin, obgleich die Häufigkeit schwerer Tacharrhythmien durch die Betablockade reduziert ist. Es gibt keine Evidenz, die die Routinegabe von i.v.-Betablockern präähospital oder in der Notaufnahme unterstützt. Die frühe i.v.-Betablocker-Gabe ist bei Patienten mit Hypotension oder Zeichen der Stauungsinsuffizienz kontraindiziert. Sie kann bei fehlenden Kontraindikationen in speziellen Situationen wie schwerer Hypertonie oder Tachyarrhythmien indiziert sein. Es ist sinnvoll, eine niedrig dosierte orale Betablocker-Therapie erst nach Stabilisierung des Patienten zu beginnen.

\section{Andere Antiarrhythmika}

Unabhängig von Betablockern gibt es keine Daten, die den Einsatz einer antiarrhythmischen Prophylaxe nach einem ACS unterstützen. Kammerflimmern (VF) verursacht die meisten frühen Todesfälle bei ACS; die Inzidenz von VF ist in den ersten Stunden nach Symptombeginn am höchsten. Dies erklärt, warum zahlreiche Studien mit dem Ziel durchgeführt wurden, den prophylaktischen Effekt einer antiarrhythmischen Therapie nachzuweisen [175]. Die Wirkungen von Antiarrhythmika (Lidocain, Magnesium, Disopyramid, Mexiletin, Verapamil, Sotalol, Tocainamid), prophylaktisch bei Patienten mit ACS gegeben, wurden untersucht. Die Prophylaxe mit Lidocain verminderte das Auftreten von VF, erhöhte aber tendenziell die Sterblichkeit [177]. Die Routinebehandlung mit Magnesium reduziert die Sterblichkeit nicht. Die Arrhythmieprophylaxe mit Disopyramid, Mexiletin, Verapamil oder anderen Antiarrhythmika, in den ersten Stunden eines ACS gegeben, beinflusst die Sterblichkeit nicht. Die Prophylaxe mit Antiarrhythmika wird deshalb nicht empfohlen.

\section{Inhibitoren des angiotensin-} konvertierenden Enzyms (ACE-Hemmer) und Angiotensin-Rezeptorblocker (ARBs)

Orale ACE-Inhibitoren verringern die Sterblichkeit, wenn sie Patienten mit Infarkt mit und ohne frühe Reperfusionstherapie gegeben werden. Die günstigen Effekte sind am deutlichsten bei Patienten mit Vorderwandinfarkt, Lungenstauung oder einer linksventrikulären Ejektionsfraktion $<40 \%$. Bei systolischem Blutdruck $<100 \mathrm{mmHg}$ zum Zeitpunkt der Aufnahme oder bei bekannten Kontraindikationen gegenüber dieser Substanzklasse, sollen ACE-Inhibitoren nicht gegeben werden. Eine höhere Sterblichkeit wurde dokumentiert, wenn eine intravenöse ACE-Inhibitor-Behandlung innerhalb der ersten $24 \mathrm{~h}$ nach Symptombeginn begonnen wurde. Eine orale ACEHemmer-Therapie ist sicher, gut verträglich und geht mit einer kleinen, aber signifikanten Sterblichkeitsreduktion einher [178]. Die Therapie soll deshalb in- nerhalb der ersten $24 \mathrm{~h}$ nach Symptombeginn begonnen werden, unabhängig davon, ob eine Reperfusionstherapie geplant ist, insbesondere bei Patienten mit Vorderwandinfarkt, Lungenstauung oder einer linksventrikulären Ejektionsfraktion unter $40 \%$. ACE-Inhibitoren sollen nicht innerhalb der ersten $24 \mathrm{~h}$ nach Symptombeginn intravenös gegeben werden $[177,179]$. Ein Angiotension-Rezeptorblocker (ARB) solle bei Patienten mit Unverträglichkeit von ACE-Inhibitoren gegeben werden [180].

\section{Lipidesenkende Behandlung}

Statine reduzieren das Auftreten wesentlicher unerwünschter kardiovaskulärer Ereignisse (MACE), wenn sie früh in den ersten Tagen nach Beginn eines ACS gegeben werden [181, 182]. Der Beginn einer Statintherapie soll bei allen Patienten innerhalb von $24 \mathrm{~h}$ nach Symptombeginn eines ACS erwogen werden. Wenn Patienten bereits Statine erhalten, soll die Therapie nicht abgebrochen werden [183].

\section{Korrespondenzadresse}

\section{N.I. Nikolaou}

Cardiology Department

Konstantopouleio General Hospital, Athens

HRArntz@t-online.de

\section{Korrespondierender Übersetzer}

\section{Prof. Dr. H.R. Arntz}

Department Emergency Medicine,

Charité, University Medicine Berlin

Campus Benjamin Franklin

Berlin, Germany

HRArntz@t-online.de

\section{Einhaltung ethischer Richtlinien}

Interessenkonflikt. N.I. Nikolaou erhält Forschungsgelder von Fourier trial-AMGEN; A. Cariou ist Ehrensprecher von BARD-France; F. Beygui ist Ehrensprecher von Astra Zeneca, Lilly, Daichi-Sankyo; A. Bellou, H.-R. Arntz und L.L. Bossaert geben an, dass kein Interessenkonflikt besteht.

Dieser Beitrag beinhaltet keine Studien an Menschen oder Tieren. 


\section{Literatur}

1. Perk J, De Backer G, Gohlke H et al (2012) European Guidelines on cardiovascular disease prevention in clinical practice (version 2012). The Fifth Joint Task Force of the European Society of Cardiology and Other Societies on Cardiovascular Disease Prevention in Clinical Practice (constituted by representatives of nine societies and by invited experts). Eur Heart J 33:1635-1701

2. Goodman SG, Huang W, Yan AT et al (2009) The expanded Global Registry of Acute Coronary Events: baseline characteristics, management practices, and hospital outcomes of patients with acute coronary syndromes. Am Heart J 158:193201 e1-5

3. Dudas K, Lappas G, Stewart S, Rosengren A (2011) Trends in out-of-hospital deaths due to coronary heart disease in Sweden (1991 to 2006). Circulation 123:46-52

4. Thygesen K, Alpert JS, Jaffe AS et al (2012) Third universal definition of myocardial infarction. $J$ Am Coll Cardiol 60:1581-1598

5. Task Force on the management of STseamiotESoC, Steg PG, James SK et al (2012) ESC Guidelines for the management of acute myocardial infarction in patients presenting with ST-segment elevation. Eur Heart J 33:2569-2619

6. Roffi M (2015) Guidelines for the diagnosis and treatment of actute coronary syndromes with and without ST-segment elevation. Circulation (im Druck)

7. Henrikson CA, Howell EE, Bush DE et al (2003) Chest pain relief by nitroglycerin does not predict active coronary artery disease. Ann Intern Med 139:979-986

8. American College of Emergency P, Society for Cardiovascular A, Interventions et al (2013) 2013 ACCF/AHA guideline for the management of STelevation myocardial infarction: a report of the American College of Cardiology Foundation/ American Heart Association Task Force on Practice Guidelines. J Am Coll Cardiol 61:e78-e140

9. Amsterdam EA, Wenger NK, Brindis RG, et al (2014) 2014 AHA/ACC guideline for the management of patients with non-ST-elevation acute coronary syndromes: executive summary: a report of the American College of Cardiology/American Heart Association Task Force on Practice Guidelines. Circulation 130:2354-2394

10. Amsterdam EA, Wenger NK, Brindis RG et al (2014) 2014 AHA/ACC Guideline for the Management of Patients with Non-ST-Elevation Acute Coronary Syndromes: a report of the American College of Cardiology/American Heart Association Task Force on Practice Guidelines. J Am Coll Cardiol 64:e139-e228

11. Canto JG, Rogers WJ, Bowlby LJ, French WJ, Pearce DJ, Weaver WD (1997) The prehospital electrocardiogram in acute myocardial infarction: is its full potential being realized? National Registry of Myocardial Infarction 2 Investigators. J Am Coll Cardiol 29:498-505

12. Terkelsen CJ, Lassen JF, Norgaard BL et al (2005) Reduction of treatment delay in patients with STelevation myocardial infarction: impact of prehospital diagnosis and direct referral to primary percutanous coronary intervention. Eur Heart J 26:770-777

13. Carstensen S, Nelson GC, Hansen PS et al (2007) Field triage to primary angioplasty combined with emergency department bypass reduces treatment delays and is associated with improved outcome. Eur Heart J 28:2313-2319
14. Brown JP, Mahmud E, Dunford JV, Ben-Yehuda O (2008) Effect of prehospital 12-lead electrocardiogram on activation of the cardiac catheterization laboratory and door-to-balloon time in STsegment elevation acute myocardial infarction. Am J Cardiol 101:158-161

15. Martinoni A, De Servi S, Boschetti E et al (2011) Importance and limits of pre-hospital electrocardiogram in patients with ST elevation myocardial infarction undergoing percutaneous coronary angioplasty. Eur J Cardiovasc Prev Rehabil 18:526-532

16. Sorensen JT, Terkelsen CJ, Norgaard BL et al (2011) Urban and rural implementation of prehospital diagnosis and direct referral for primary percutaneous coronary intervention in patients with acute ST-elevation myocardial infarction. Eur Heart J 32:430-436

17. Chan AW, Kornder J, Elliott $\mathrm{H}$ et al (2012) Improved survival associated with pre-hospital triage strategy in a large regional ST-segment elevation myocardial infarction program. JACC CardiovasC Interv 5:1239-1246

18. Quinn T, Johnsen S, Gale CP et al (2014) Effects of prehospital 12-lead ECG on processes of care and mortality in acute coronary syndrome: a linked cohort study from the Myocardial Ischaemia National Audit Project. Heart 100:944-950

19. Ong ME, Wong AS, Seet CM et al (2013) Nationwide improvement of door-to-balloon times in patients with acute ST-segment elevation myocardial infarction requiring primary percutaneous coronary intervention with out-of-hospital 12-lead ECG recording and transmission. Ann Emerg Med 61:339-347

20. Swor R, Hegerberg S, McHugh-McNally A, Goldstein M, McEachin CC (2006) Prehospital 12-lead ECG: efficacy or effectiveness? Prehosp Emerg Care 10:374-377

21. Masoudi FA, Magid DJ, Vinson DR et al (2006) Implications of the failure to identify high-risk electrocardiogram findings for the quality of care of patients with acute myocardial infarction: results of the Emergency Department Quality in Myocardial Infarction (EDQMI) study. Circulation 114:1565-1571

22. Kudenchuk PJ, Ho MT, Weaver WD et al (1991) Accuracy of computer-interpreted electrocardiography in selecting patients for thrombolytic therapy. MITI Project Investigators. J Am Coll Cardiol 17:1486-1491

23. Dhruva VN, Abdelhadi SI, Anis A et al (2007) STSegment Analysis Using Wireless Technology in Acute Myocardial Infarction (STAT-MI) trial. J Am Coll Cardiol 50:509-513

24. Bhalla MC, Mencl F, Gist MA, Wilber S, Zalewski J (2013) Prehospital electrocardiographic computer identification of ST-segment elevation myocardial infarction. Prehosp Emerg Care 17:211216

25. Clark EN, Sejersten M, Clemmensen P, Macfarlane PW (2010) Automated electrocardiogram interpretation programs versus cardiologists' triage decision making based on teletransmitted data in patients with suspected acute coronary syndrome. Am J Cardiol 106:1696-1702

26. de Champlain F, Boothroyd LJ, Vadeboncoeur A et al (2014) Computerized interpretation of the prehospital electrocardiogram: predictive value for ST segment elevation myocardial infarction and impact on on-scene time. CJEM 16:94-105
27. Squire BT, Tamayo-Sarver JH, Rashi P, Koenig W, Niemann JT (2014) Effect of prehospital cardiac catheterization lab activation on door-to-balloon time, mortality, and false-positive activation. Prehosp Emerg Care 18:1-8

28. Youngquist ST, Shah AP, Niemann JT, Kaji AH, French WJ (2008) A comparison of door-to-balloon times and false-positive activations between emergency department and out-of-hospital activation of the coronary catheterization team. Acad Emerg Med 15:784-787

29. van't Hof AW, Rasoul $S$, van de Wetering $\mathrm{H}$ et al (2006) Feasibility and benefit of prehospital diagnosis, triage, and therapy by paramedics only in patients who are candidates for primary angioplasty for acute myocardial infarction. Am Heart 151:1255e1-5

30. Layfield C, Rose J, Alford A et al (2015) Effectiveness of practices for improving the diagnostic accuracy of Non ST Elevation Myocardial Infarction in the Emergency Department: a laboratory medicine best practices systematic review. Clin Biochem 48:204-212

31. Hamm CW, Bassand JP, Agewall S et al (2011) ESC Guidelines for the management of acute coronary syndromes in patients presenting without persistent ST-segment elevation: The Task Force for the management of acute coronary syndromes (ACS) in patients presenting without persistent ST-segment elevation of the European Society of Cardiology (ESC). Eur Heart J 32:2999-3054

32. Keller T, Zeller T, Peetz D et al (2009) Sensitive troponin I assay in early diagnosis of acute myocardial infarction. N Engl J Med 361:868-877

33. Pope JH, Aufderheide TP, Ruthazer R et al (2000) Missed diagnoses of acute cardiac ischemia in the emergency department. N Engl J Med 342:1163-1170

34. Collinson PO, Premachandram S, Hashemi K (2000) Prospective audit of incidence of prognostically important myocardial damage in patients discharged from emergency department. BMJ 320:1702-1705

35. Aldous SJ, Richards M, Cullen L, Troughton R, Than M (2012) A 2-hour thrombolysis in myocardial infarction score outperforms other risk stratification tools in patients presenting with possible acute coronary syndromes: comparison of chest pain risk stratification tools. Am Heart J 164:516523

36. Than M, Cullen L, Reid CM et al (2011) A 2-h diagnostic protocol to assess patients with chest pain symptoms in the Asia-Pacific region (ASPECT): a prospective observational validation study. Lancet 377:1077-1084

37. Than M, Cullen L, Aldous S et al (2012) 2-Hour ac celerated diagnostic protocol to assess patients with chest pain symptoms using contemporary troponins as the only biomarker: the ADAPT trial. J Am Coll Cardiol 59:2091-2098

38. Reichlin T, Schindler C, Drexler B et al (2012) Onehour rule-out and rule-in of acute myocardial infarction using high-sensitivity cardiac troponin T. Arch Intern Med 172:1211-1218

39. Meller B, Cullen L, Parsonage WA et al (2015) Accelerated diagnostic protocol using high-sensitivity cardiac troponin $\mathrm{T}$ in acute chest pain patients. Int J Cardiol 184:208-215

40. Nikolaou N, Welsford M, Beygui F et al (2015) Part 5: acute coronary syndromes: 2015 International Consensus on Cardiopulmonary Resuscitation and Emergency Cardiovascular Care Science With Treatment Recommendations. Resuscitation (im Druck) 
41. Renaud B, Maison P, Ngako A et al (2008) Impact of point-of-care testing in the emergency department evaluation and treatment of patients with suspected acute coronary syndromes. Acad Emerg Med 15:216-224

42. Mitchell AM, Garvey JL, Kline JA (2006) Multimarker panel to rule out acute coronary syndromes in low-risk patients. Acad Emerg Med 13:803806

43. Sorensen JT, Terkelsen CJ, Steengaard C et al (2011) Prehospital troponin T testing in the diagnosis and triage of patients with suspected acute myocardial infarction. Am J Cardiol 107:14361440

44. Loewenstein D, Stake C, Cichon M (2013) Assessment of using fingerstick blood sample with iSTAT point-of-care device for cardiac troponin I assay. Am J Emerg Med 31:1236-1239

45. D'Ascenzo F, Biondi-Zoccai G, Moretti C et al (2012) TIMI, GRACE and alternative risk scores in acute coronary syndromes: a meta-analysis of 40 derivation studies on 216,552 patients and of 42 validation studies on 31,625 patients. Contemp Clin Trials 33:507-514

46. Cullen L, Greenslade J, Hammett CJ et al (2013) Comparison of three risk stratification rules for predicting patients with acute coronary syndrome presenting to an Australian emergency department. Heart Lung Circ 22:844-851

47. Lin A, Devlin G, Lee M, Kerr AJ (2014) Performance of the GRACE scores in a New Zealand acute coronary syndrome cohort. Heart 100:1960-1966

48. Tymchak W, Armstrong PW, Westerhout CM et al (2011) Mode of hospital presentation in patients with non-ST-elevation myocardial infarction: implications for strategic management. Am Heart J 162:436-443

49. Fox KA, Fitzgerald G, Puymirat E et al (2014) Should patients with acute coronary disease be stratified for management according to their risk? Derivation, external validation and outcomes using the updated GRACE risk score. BMJ Open 4:e004425

50. Farkouh ME, Smars PA, Reeder GS et al (1989) A clinical trial of a chest-pain observation unit for patients with unstable angina. Chest Pain Evaluation in the Emergency Room (CHEER) Investigators. N Engl J Med 339:1882-1888

51. Hermann LK, Newman DH, Pleasant WA et al (2013) Yield of routine provocative cardiac testing among patients in an emergency department-based chest pain unit. JAMA Intern Med 173:1128-1133

52. Ramakrishna G, Milavetz JJ, Zinsmeister AR et al (2005) Effect of exercise treadmill testing and stress imaging on the triage of patients with chest pain: CHEER substudy. Mayo Clin Proc 80:322-329

53. Goldstein JA, Gallagher MJ, O'Neill WW, Ross MA, O’Neil BJ, Raff GL (2007) A randomized controlled trial of multi-slice coronary computed tomography for evaluation of acute chest pain. J Am Coll Cardiol 49:863-871

54. Forberg $\mathrm{J}$, Hilmersson $\mathrm{CE}$, Carlsson $\mathrm{M}$ et al (2009) Negative predictive value and potential cost savings of acute nuclear myocardial perfusion imaging in low risk patients with suspected acute coronary syndrome: a prospective single blinded study. BMC Emerg Med 9:12
55. Nucifora G, Badano LP, Sarraf-Zadegan N et al (2007) Comparison of early dobutamine stress echocardiography and exercise electrocardiographic testing for management of patients presenting to the emergency department with chest pain. Am J Cardiol 100:1068-1073

56. Wei K (2010) Utility contrast echocardiography in the emergency department. JACC Cardiovasc Imaging 3:197-203

57. Gaibazzi N, Squeri A, Reverberi C et al (2011) Contrast stress-echocardiography predicts cardiac events in patients with suspected acute coronary syndrome but nondiagnostic electrocardiogram and normal 12-hour troponin. J Am Soc Echocardiogr 24:1333-1341

58. Douglas PS, Khandheria B, Stainback RF et al (2007) ACCF/ASE/ACEP/ASNC/SCAI/SCCT/SCMR 2007 appropriateness criteria for transthoracic and transesophageal echocardiography: a report of the American College of Cardiology Foundation Quality Strategic Directions Committee Appropriateness Criteria Working Group, American Society of Echocardiography, American College of Emergency Physicians, American Society of Nuclear Cardiology, Society for Cardiovascular Angiography and Interventions, Society of Cardiovascular Computed Tomography, and the Society for Cardiovascular Magnetic Resonance endorsed by the American College of Chest Physicians and the Society of Critical Care Medicine. J Am Coll Cardiol 50:187-204

59. Hoffmann U, Bamberg F, Chae CU et al (2009) Coronary computed tomography angiography for early triage of patients with acute chest pain: the ROMICAT (Rule Out Myocardial Infarction using Computer Assisted Tomography) trial. J Am Coll Cardiol 53:1642-1650

60. Hoffmann U, Nagurney JT, Moselewski F et al (2006) Coronary multidetector computed tomography in the assessment of patients with acute chest pain. Circulation 114:2251-2260

61. Hollander JE, Chang AM, Shofer FS, McCusker CM, Baxt WG, Litt HI (2009) Coronary computed tomographic angiography for rapid discharge of low-risk patients with potential acute coronary syndromes. Ann Emerg Med 53:295-304

62. Pundziute G, Schuijf JD, Jukema JW et al (2007) Prognostic value of multislice computed tomography coronary angiography in patients with known or suspected coronary artery disease. J Am Coll Cardiol 49:62-70

63. Rubinshtein R, Halon DA, Gaspar T et al (2007) Impact of 64-slice cardiac computed tomographic angiography on clinical decision-making in emergency department patients with chest pain of possible myocardial ischemic origin. Am J Cardiol 100:1522-1526

64. Janne d'Othee B, Siebert U, Cury R, Jadvar H, Dunn EJ, Hoffmann U (2008) A systematic review on diagnostic accuracy of CT-based detection of significant coronary artery disease. Eur J Radiol 65:449-461

65. Sirol M, Sanz J, Henry P, Rymer R, Leber A (2009) Evaluation of 64-slice MDCT in the real world of cardiology: a comparison with conventional coronary angiography. Arch Cardiovasc Dis 102:433-449

66. Galperin-Aizenberg M, Cook TS, Hollander JE Litt HI (2015) Cardiac CT angiography in the emergency department. AJR Am J Roentgenol 204:463-474
67. Cury RC, Feuchtner GM, Batlle JC et al (2013) Triage of patients presenting with chest pain to the emergency department: implementation of coronary $\mathrm{CT}$ angiography in a large urban health care system. AJR Am J Roentgenol 200:57-65

68. Gruettner J, Henzler T, Sueselbeck T, Fink C, Borggrefe M, Walter T (2012) Clinical assessment of chest pain and guidelines for imaging. Eur J Radiol 81:3663-3668

69. Samad Z, Hakeem A, Mahmood SS et al (2012) A meta-analysis and systematic review of computed tomography angiography as a diagnostic triage tool for patients with chest pain presenting to the emergency department. J Nucl Cardiol 19:364-376

70. Werns SW (2007) Are nitrates safe in patients who use sildenafil? Maybe. Crit Care Med 35:1988-1990

71. O'Gara PT, Kushner FG, Ascheim DD et al (2013) 2013 ACCF/AHA guideline for the management of ST-elevation myocardial infarction: a report of the American College of Cardiology Foundation/ American Heart Association Task Force on Practice Guidelines. Circulation 127:e362-e425

72. Kearney PM, Baigent C, Godwin J, Halls H, Emberson JR, Patrono C (2006) Do selective cyclo-oxygenase-2 inhibitors and traditional non-steroidal anti-inflammatory drugs increase the risk of atherothrombosis? Meta-analysis of randomised trials. BMJ 332:1302-1308

73. Rawles JM, Kenmure AC (1976) Controlled trial of oxygen in uncomplicated myocardial infarction. Br Med J 1:1121-1123

74. Wijesinghe $M$, Perrin $K$, Ranchord $A$, Simmonds M, Weatherall M, Beasley R (2009) Routine use of oxygen in the treatment of myocardial infarction: systematic review. Heart 95:198-202

75. Stub D, Smith K, Bernard S et al (2015) Air versus oxygen in ST-segment elevation myocardial infarction. Circulation 131(24):2143-2150

76. Cabello JB, Burls A, Emparanza Jl, Bayliss S, Quinn T (2013) Oxygen therapy for acute myocardial infarction. Cochrane Database Syst Rev 8:CD007160

77. Nolan JP, Soar J, Cariou A et al (2015) European Resuscitation Council Guidelines for Resuscitation 2015 Section 5 Post Resuscitation Care. Resuscitation (im Druck)

78. Freimark D, Matetzky S, Leor J et al (2002) Timing of aspirin administration as a determinant of survival of patients with acute myocardial infarction treated with thrombolysis. Am J Cardiol 89:381385

79. Frilling B, Schiele R, Gitt AK et al (2001) Characterization and clinical course of patients not receiving aspirin for acute myocardial infarction: results from the MITRA and MIR studies. Am Heart 141:200-205

80. O'Gara PT, Kushner FG, Ascheim DD et al (2013) 2013 ACCF/AHA guideline for the management of ST-elevation myocardial infarction: executive summary: a report of the American College of Cardiology Foundation/American Heart Association Task Force on Practice Guidelines. Circulation 127:529-555

81. Wiviott SD, Braunwald E, McCabe CH et al (2007) Prasugrel versus clopidogrel in patients with acute coronary syndromes. N Engl J Med 357:20012015

82. Wallentin L, Becker RC, Budaj A et al (2009) Ticagrelor versus clopidogrel in patients with acute coronary syndromes. N Engl J Med 361:1045-1057 
83. Yusuf S, Zhao F, Mehta SR, Chrolavicius S, Tognoni G, Fox KK (2001) Effects of clopidogrel in addition to aspirin in patients with acute coronary syndromes without ST-segment elevation. N Engl J Med 345:494-502

84. Montalescot G, Bolognese L, Dudek D et al (2013) Pretreatment with prasugrel in non-ST-segment elevation acute coronary syndromes. N Engl J Med 369:999-1010

85. Zeymer U (2013) Oral antiplatelet therapy in acute coronary syndromes: recent developments. Cardiol Ther 2:47-56

86. Ducci K, Grotti S, Falsini G et al (2013) Comparison of pre-hospital $600 \mathrm{mg}$ or $900 \mathrm{mg}$ vs. peri-interventional $300 \mathrm{mg}$ clopidogrel in patients with ST-elevation myocardial infarction undergoing primary coronary angioplasty. The Load \& Go randomized trial. Int J Cardiol 168:4814-4816

87. Bellemain-Appaix A, O'Connor SA, Silvain J et al (2012) Association of clopidogrel pretreatment with mortality, cardiovascular events, and major bleeding among patients undergoing percutacoronary intervention: a systematic review and meta-analysis. JAMA 308:2507-2516

88. Chen ZM, Jiang LX, Chen YP et al (2005) Addition of clopidogrel to aspirin in 45,852 patients with acute myocardial infarction: randomised placebo-controlled trial. Lancet 366:1607-1621

89. Sabatine MS, Cannon CP, Gibson CM et al (2005) Addition of clopidogrel to aspirin and fibrinolytic therapy for myocardial infarction with ST-segment elevation. N Engl J Med 352:1179-1189

90. Verheugt FW, Montalescot G, Sabatine MS et al (2007) Prehospital fibrinolysis with dual antiplatelet therapy in ST-elevation acute myocardial infarction: a substudy of the randomized double blind CLARITY-TIMI 28 trial. J Thromb Thrombolysis 23:173-179

91. Montalescot G, Wiviott SD, Braunwald E et al (2009) Prasugrel compared with clopidogrel in patients undergoing percutaneous coronary intervention for ST-elevation myocardial infarction (TRITON-TIMI 38): double-blind, randomised controlled trial. Lancet 373:723-731

92. Montalescot G, van 't Hof AW, Lapostolle F et al (2014) Prehospital ticagrelor in ST-segment elevation myocardial infarction. $\mathrm{N}$ Engl J Med 371:1016-1027

93. Boersma E, Harrington RA, Moliterno DJ et al (2002) Platelet glycoprotein Ilb/llla inhibitors in acute coronary syndromes: a meta-analysis of all major randomised clinical trials. [erratum appears in Lancet 2002 Jun 15;359(9323):2120]. Lancet 359:189-198

94. Keeley EC, Boura JA, Grines CL (2006) Comparison of primary and facilitated percutaneous coronary interventions for ST-elevation myocardial infarction: quantitative review of randomised trials. Lancet 367:579-588

95. ten Berg JM, van 't Hof AW, Dill T et al (2010) Effect of early, pre-hospital initiation of high bolus dose tirofiban in patients with ST-segment elevation myocardial infarction on short- and longterm clinical outcome. J Am Coll Cardiol 55:24462455

96. DE Luca G, Bellandi F, Huber K et al (2011) Early glycoprotein Ilb-Illa inhibitors in primary angioplasty-abciximab long-term results (EGYPT-ALT) cooperation: individual patient's data meta-analysis. J Thromb Haemost 9:2361-2370
97. Mehilli J, Kastrati A, Schulz S et al (2009) Abciximab in patients with acute ST-segment-elevation myocardial infarction undergoing primary percutaneous coronary intervention after clopidogrel loading: a randomized double-blind trial. Circulation 119:1933-1940

98. Steg PG, van 't Hof A, Hamm CW et al (2013) Bivalirudin started during emergency transport for primary PCI. N Engl J Med 369:2207-2217

99. Montalescot G, Zeymer U, Silvain J et al (2011) Intravenous enoxaparin or unfractionated heparin in primary percutaneous coronary intervention for ST-elevation myocardial infarction: the international randomised open-label ATOLL trial. Lancet 378:693-703

100. Sejersten $M$, Nielsen SL, Engstrom T, Jorgensen E, Clemmensen P (2009) Feasibility and safety of prehospital administration of bivalirudin in patients with ST-elevation myocardial infarction. Am J Cardiol 103:1635-1640

101. Mega JL, Braunwald E, Wiviott SD et al (2012) Rivaroxaban in patients with a recent acute coronary syndrome. N Engl J Med 366:9-19

102. TIMI-11B Investigators, Antman EM, McCabe CH et al (1999) Enoxaparin prevents death and cardiac ischemic events in unstable angina/non-Qwave myocardial infarction. Results of the thrombolysis in myocardial infarction (TIMI) 11B trial. Circulation 100:1593-1601

103. Cohen M, Demers C, Gurfinkel EP et al (1997) A comparison of low-molecular-weight heparin with unfractionated heparin for unstable coronary artery disease. Efficacy and Safety of Subcutaneous Enoxaparin in Non-Q-Wave Coronary Events Study Group. N Engl J Med 337:447-452

104. Moscucci M, Fox KA, Cannon CP et al (2003) Predictors of major bleeding in acute coronary syndromes: the Global Registry of Acute Coronary Events (GRACE). Eur Heart J 24:1815-1823

105. Yusuf S, Mehta SR, Chrolavicius S et al (2006) Comparison of fondaparinux and enoxaparin in acute coronary syndromes. N Engl J Med 354:1464-1476

106. Mehta SR, Boden WE, Eikelboom JW et al (2008) Antithrombotic therapy with fondaparinux in relation to interventional management strategy in patients with ST- and non-ST-segment elevation acute coronary syndromes: an individual patientlevel combined analysis of the Fifth and Sixth Organization to Assess Strategies in Ischemic Syndromes (OASIS5 and 6) randomized trials. Circulation 118:2038-2046

107. Stone GW, McLaurin BT, Cox DA et al (2006) Bivalirudin for patients with acute coronary syndromes. N Engl J Med 355:2203-2216

108. Ferguson JJ, Califf RM, Antman EM et al (2004) Enoxaparin vs unfractionated heparin in highrisk patients with non-ST-segment elevation acute coronary syndromes managed with an intended early invasive strategy: primary results of the SYNERGY randomized trial. JAMA 292:45-54

109. Wallentin L, Goldstein P, Armstrong PW et al (2003) Efficacy and safety of tenecteplase in combination with the low-molecular-weight heparin enoxaparin or unfractionated heparin in the prehospital setting: the Assessment of the Safety and Efficacy of a New Thrombolytic Regimen (ASSENT)-3 PLUS randomized trial in acute myocardial infarction. Circulation 108:135-142

110. Antman EM, Morrow DA, McCabe CH et al (2006) Enoxaparin versus unfractionated heparin with fibrinolysis for ST-elevation myocardial infarction. N Engl J Med 354:1477-1488
111. Hirschl MM, Mayr H, Erhart F et al (2012) Prehospital treatment of patients with acute myocardial infarction with bivalirudin. Am J Emerg Med 30:12-17

112. Zijlstra F, Ernst N, De Boer M-J et al (2002) Influence of prehospital administration of aspirin and heparin on initial patency of the infarct-related artery in patients with acute ST elevation myocardial infarction. J Am Coll Cardiol 39:17331737

113. Zeymer U, Gitt A, Zahn R et al (2009) Efficacy and safety of enoxaparin in combination with and without GP Ilb/Illa inhibitors in unselected patients with ST segment elevation myocardial infarction treated with primary percutaneous coronary intervention. Eurolntervention 4:524-528

114. Stone GW, Witzenbichler B, Guagliumi $G$ et al (2008) Bivalirudin during primary $\mathrm{PCl}$ in acute myocardial infarction. N Engl J Med 358:22182230

115. Mehran R, Lansky AJ, Witzenbichler B et al (2009) Bivalirudin in patients undergoing primary angioplasty for acute myocardial infarction (HORIZONS-AMI): 1-year results of a randomised controlled trial. Lancet 374:1149-1159

116. Schulz S, Richardt G, Laugwitz KL et al (2014) Prasugrel plus bivalirudin vs. clopidogrel plus heparin in patients with ST-segment elevation myocardial infarction. Eur Heart J 35:2285-2294

117. Shahzad A, Kemp I, Mars C et al (2014) Unfractionated heparin versus bivalirudin in primary percutaneous coronary intervention (HEAT-PPCI): an open-label, single centre, randomised controlled trial. Lancet 384:1849-1858

118. White HD, Aylward PE, Frey MJ et al (1997) Randomized, double-blind comparison of hirulog versus heparin in patients receiving streptokinase and aspirin for acute myocardial infarction (HERO). Hirulog Early Reperfusion/Occlusion (HERO) Trial Investigators. Circulation 96:2155161

119. Antman EM, Anbe DT, Armstrong PW et al (2004) ACC/AHA guidelines for the management of patients with ST-elevation myocardial infarction - executive summary: a report of the American College of Cardiology/American Heart Association Task Force on Practice Guidelines (Writing Committee to Revise the 1999 Guidelines for the Management of Patients With Acute Myocardial Infarction). Circulation 110:588-636

120. Bassand JP, Hamm CW, Ardissino D et al (2007) Guidelines for the diagnosis and treatment of non-ST-segment elevation acute coronary syndromes. Eur Heart J 28:1598-1660

121. Anderson JL, Adams CD, Antman EM et al (2007) ACC/AHA 2007 guidelines for the management of patients with unstable angina/non ST-elevation myocardial infarction: a report of the American College of Cardiology/American Heart Association Task Force on Practice Guidelines (Writing Committee to Revise the 2002 Guidelines for the Management of Patients With Unstable Angina/ Non ST-Elevation Myocardial Infarction): developed in collaboration with the American College of Emergency Physicians, the Society for Cardiovascular Angiography and Interventions, and the Society of Thoracic Surgeons: endorsed by the American Association of Cardiovascular and Pulmonary Rehabilitation and the Society for Academic Emergency Medicine. Circulation 116:e148e304 
122. Kushner FG, Hand M, Smith SC Jr et al (2009) 2009 Focused Updates: ACC/AHA Guidelines for the Management of Patients With ST-Elevation Myocardial Infarction (updating the 2004 Guideline and 2007 Focused Update) and ACC/AHA/ SCAI Guidelines on Percutaneous Coronary Intervention (updating the 2005 Guideline and 2007 Focused Update): a report of the American College of Cardiology Foundation/American Heart Association Task Force on Practice Guidelines. Circulation 120:2271-2306. (Erratum in: Circulation. 010 Mar 30;121(12):e257. Dosage error in article text)

123. Castaigne AD, Herve C, Duval-Moulin AM et al (1989) Prehospital use of APSAC: results of a placebo-controlled study. Am J Cardiol 64:30A-3A (discussion 41A-2A)

124. Schofer J, Buttner J, Geng G et al (1990) Prehospital thrombolysis in acute myocardial infarction. Am J Cardiol 66:1429-1433

125. Weaver WD, Cerqueira M, Hallstrom AP et al (1993) Prehospital-initiated vs hospital-initiated thrombolytic therapy. The Myocardial Infarction Triage and Intervention Trial. JAMA 270:12111216

126. Welsh RC, Travers A, Senaratne M, Williams R, Armstrong PW (2006) Feasibility and applicability of paramedic-based prehospital fibrinolysis in a large North American center. Am Heart J 152:1007-1014

127. Armstrong PW, Gershlick AH, Goldstein P et al (2013) Fibrinolysis or primary PCl in ST-segment elevation myocardial infarction. N Engl J Med 368:1379-1387

128. Larson DM, Duval S, Sharkey SW et al (2012) Safety and efficacy of a pharmaco-invasive reperfusion strategy in rural ST-elevation myocardial infarction patients with expected delays due to long-distance transfers. Eur Heart J 33:12321240

129. Keeley EC, Boura JA, Grines CL (2003) Primary angioplasty versus intravenous thrombolytic therapy for acute myocardial infarction: a quantitative review of 23 randomised trials. Lancet 361:13-20

130. Bonnefoy E, Steg PG, Boutitie F et al (2009) Comparison of primary angioplasty and pre-hospital fibrinolysis in acute myocardial infarction (CAPTIM) trial: a 5-year follow-up. Eur Heart J 30:1598-1606

131. Kalla K, Christ G, Karnik R et al (2006) Implementation of guidelines improves the standard of care: the Viennese registry on reperfusion strategies in ST-elevation myocardial infarction (Vienna STEMI registry). Circulation 113:2398-2405

132. Terkelsen CJ, Sorensen JT, Maeng M et al (2010) System delay and mortality among patients with STEMI treated with primary percutaneous coronary intervention. JAMA 304:763-771

133. Pinto DS, Kirtane AJ, Nallamothu BK et al (2006) Hospital delays in reperfusion for ST-elevation myocardial infarction: implications when selecting a reperfusion strategy. Circulation 114:20192025

134. Madsen MM, Busk M, Sondergaard HM et al (2005) Does diabetes mellitus abolish the beneficial effect of primary coronary angioplasty on long-term risk of reinfarction after acute ST-segment elevation myocardial infarction compared with fibrinolysis? (A DANAMI-2 substudy). Am J Cardiol 96:1469-1475

135. Le May MR, So DY, Dionne R et al (2008) A citywide protocol for primary PCI in ST-segment elevation myocardial infarction. N Engl J Med 358:231-240
136. Bradley EH, Herrin J, Wang Y et al (2006) Strategies for reducing the door-to-balloon time in acute myocardial infarction. $N$ Engl J Med 355:2308-2320

137. Bonnefoy $E$, Lapostolle $F$, Leizorovicz A et al (2002) Primary angioplasty versus prehospital fibrinolysis in acute myocardial infarction: a randomised study. Lancet 360:825-829

138. Armstrong PW (2006) A comparison of pharmacologic therapy with/without timely coronary intervention vs. primary percutaneous intervention early after ST-elevation myocardial infarction: the WEST (Which Early ST-elevation myocardial infarction Therapy) study. Eur Heart J 27:1530-1538

139. Thiele H, Eitel I, Meinberg C et al (2011) Randomized comparison of pre-hospital-initiated facilitated percutaneous coronary intervention versus primary percutaneous coronary intervention in acute myocardial infarction very early after symptom onset: the LIPSIA-STEMI trial (Leipzig immediate prehospital facilitated angioplasty in STsegment myocardial infarction). JACC Cardiovasc Interv 4:605-614

140. Andersen $H R$, Nielsen TT, Rasmussen $K$ et al (2003) A comparison of coronary angioplasty with fibrinolytic therapy in acute myocardial infarction. N Engl J Med 349:733-742

141. Dieker HJ, van Horssen EV, Hersbach FM et al (2006) Transport for abciximab facilitated primary angioplasty versus on-site thrombolysis with a liberal rescue policy: the randomised Holland Infarction Study (HIS). J Thromb Thrombolysis 22:39-45

142. Dobrzycki S, Kralisz P, Nowak K et al (2007) Trans fer with GP IIb/Illa inhibitor tirofiban for primary percutaneous coronary intervention vs. on-site thrombolysis in patients with ST-elevation myocardial infarction (STEMI): a randomized openlabel study for patients admitted to community hospitals. Eur Heart J 28:2438-2448

143. Grines CL, Westerhausen DR Jr, Grines LL et al (2002) A randomized trial of transfer for primary angioplasty versus on-site thrombolysis in patients with high-risk myocardial infarction: the Air Primary Angioplasty in Myocardial Infarction study. J Am Coll Cardiol 39:1713-1719

144. Svensson L, Aasa M, Dellborg M et al (2006) Comparison of very early treatment with either fibrinolysis or percutaneous coronary intervention facilitated with abciximab with respect to ST recovery and infarct-related artery epicardial flow in patients with acute ST-segment elevation myocardial infarction: the Swedish Early Decision (SWEDES) reperfusion trial. Am Heart J 151:798 e1-7

145. Vermeer F, Oude Ophuis AJ, vd Berg EJ et al (1999) Prospective randomised comparison between thrombolysis, rescue PTCA, and primary PTCA in patients with extensive myocardial infarction admitted to a hospital without PTCA facilities: a safety and feasibility study. Heart 82:426-431

146. Widimsky P, Groch L, Zelizko M, Aschermann M, Bednar F, Suryapranata H (2000) Multicentre randomized trial comparing transport to primary angioplasty vs immediate thrombolysis vs combined strategy for patients with acute myocardial infarction presenting to a community hospital without a catheterization laboratory. The PRAGUE study. Eur Heart J 21:823-831
147. Widimsky P, Budesinsky T, Vorac D et al (2003) Long distance transport for primary angioplasty vs immediate thrombolysis in acute myocardial infarction. Final results of the randomized national multicentre trial-PRAGUE-2. Eur Heart J 24:94-104

148. Van de Werf F, Barron HV, Armstrong PW et al (2001) Incidence and predictors of bleeding events after fibrinolytic therapy with fibrin-specific agents: a comparison of TNK-tPA and rt-PA. Eur Heart J 22:2253-2261

149. Ellis SG, Tendera M, de Belder MA et al (2008) Facilitated $\mathrm{PCl}$ in patients with ST-elevation myocardial infarction. N Engl J Med 358:2205-2217

150. Itoh T, Fukami K, Suzuki T et al (2010) Comparison of long-term prognostic evaluation between pre-intervention thrombolysis and primary coronary intervention: a prospective randomized trial: five-year results of the IMPORTANT study. Circ J 74:1625-1634

151. Kurihara $H$, Matsumoto S, Tamura R et al (2004) Clinical outcome of percutaneous coronary intervention with antecedent mutant t-PA administration for acute myocardial infarction. Am Heart J 147:E14

152. Thiele H, Scholz M, Engelmann L et al (2006) STsegment recovery and prognosis in patients with ST-elevation myocardial infarction reperfused by prehospital combination fibrinolysis, prehospital initiated facilitated percutaneous coronary intervention, or primary percutaneous coronary intervention. Am J Cardiol 98:1132-1139

153. Gershlick AH, Stephens-Lloyd A, Hughes S et al (2005) Rescue angioplasty after failed thrombolytic therapy for acute myocardial infarction. $\mathrm{N}$ Engl J Med 353:2758-2768

154. Scheller B, Hennen B, Hammer B et al (2003) Beneficial effects of immediate stenting after thrombolysis in acute myocardial infarction. J Am Coll Cardiol 42:634-641

155. Fernandez-Aviles F, Alonso JJ, Castro-Beiras A et al (2004) Routine invasive strategy within 24 hours of thrombolysis versus ischaemia-guided conservative approach for acute myocardial infarction with ST-segment elevation (GRACIA-1): a randomised controlled trial. Lancet 364:10451053

156. Le May MR, Wells GA, Labinaz M et al (2005) Combined angioplasty and pharmacological intervention versus thrombolysis alone in acute myocardial infarction (CAPITAL AMI study). J Am Coll Cardiol 46:417-424

157. Cantor WJ, Fitchett D, Borgundvaag B et al (2009) Routine early angioplasty after fibrinolysis for acute myocardial infarction. N Engl J Med 360:2705-2718

158. Bohmer E, Hoffmann $P$, Abdelnoor $M$, Arnesen $H$, Halvorsen S (2010) Efficacy and safety of immediate angioplasty versus ischemia-guided management after thrombolysis in acute myocardial infarction in areas with very long transfer distances results of the NORDISTEMI (NORwegian study on District treatment of ST-elevation myocardial infarction). J Am Coll Cardiol 55:102-110

159. Fernandez-Aviles F, Alonso JJ, Pena G et al (2007) Primary angioplasty vs. early routine post-fibrinolysis angioplasty for acute myocardial infarction with ST-segment elevation: the GRACIA-2 non-inferiority, randomized, controlled trial. Eur Heart J 28:949-960 
160. Danchin N, Coste P, Ferrieres J et al (2008) Comparison of thrombolysis followed by broad use of percutaneous coronary intervention with primary percutaneous coronary intervention for STsegment-elevation acute myocardial infarction: data from the french registry on acute ST-elevation myocardial infarction (FAST-MI). Circulation 118:268-276

161. Thiele H, Zeymer U, Neumann FJ et al (2012) Intraaortic balloon support for myocardial infarction with cardiogenic shock. N Engl J Med 367:1287-1296

162. Hochman JS, Sleeper LA, Webb JG et al (2006) Early revascularization and long-term survival in cardiogenic shock complicating acute myocardial infarction. JAMA 295:2511-2515

163. Larsen JM, Ravkilde J (2012) Acute coronary angiography in patients resuscitated from out-ofhospital cardiac arrest-a systematic review and meta-analysis. Resuscitation 83:1427-1433

164. Spaulding CM, Joly LM, Rosenberg A et al (1997) Immediate coronary angiography in survivors of out-of-hospital cardiac arrest. N Engl J Med 336:1629-1633

165. Camuglia AC, Randhawa VK, Lavi S, Walters DL (2014) Cardiac catheterization is associated with superior outcomes for survivors of out of hospital cardiac arrest: review and meta-analysis. Resuscitation 85:1533-1540

166. Rab T, Kern KB, Tamis-Holland JE et al (2015) Cardiac arrest: a treatment algorithm for emergent invasive cardiac procedures in the resuscitated comatose patient. J Am Coll Cardiol 66:62-73

167. Dumas F, Manzo-Silberman S, Fichet J et al (2012) Can early cardiac troponin I measurement help to predict recent coronary occlusion in out-ofhospital cardiac arrest survivors? Crit care Med 40:1777-1784

168. Dumas F, Cariou A, Manzo-Silberman S et al (2010) Immediate percutaneous coronary intervention is associated with better survival after out-of-hospital cardiac arrest: insights from the PROCAT (Parisian Region Out of hospital Cardiac ArresT) registry. Circ Cardiovasc Interv 3:200-207

169. Bro-Jeppesen J, Kjaergaard J, Wanscher M et al (2012) Emergency coronary angiography in comatose cardiac arrest patients: do real-life experiences support the guidelines? Eur Heart J Acute Cardiovasc Care 1:291-301

170. Hollenbeck RD, McPherson JA, Mooney MR et al (2014) Early cardiac catheterization is associated with improved survival in comatose survivors of cardiac arrest without STEMI. Resuscitation 85:88-95

171. Dankiewicz J, Nielsen N, Annborn M et al (2015) Survival in patients without acute ST elevation after cardiac arrest and association with early coronary angiography: a post hoc analysis from the TTM trial. Intensive Care Med 41:856-864

172. Noc M, Fajadet J, Lassen JF et al (2014) Invasive coronary treatment strategies for out-of-hospital cardiac arrest: a consensus statement from the European association for percutaneous cardiovascular interventions (EAPCI)/stent for life (SFL) groups. Eurolntervention 10:31-37

173. Yusuf S, Peto R, Lewis J, Collins R, Sleight $P$ (1985) Beta blockade during and after myocardial infarction: an overview of the randomized trials. Prog Cardiovasc Dis 27:335-371

174. Chen ZM, Pan HC, Chen YP et al (2005) Early intravenous then oral metoprolol in 45,852 patients with acute myocardial infarction: randomised placebo-controlled trial. Lancet 366:1622-1632
175. Teo KK, Yusuf S, Furberg CD (1993) Effects of prophylactic antiarrhythmic drug therapy in acute myocardial infarction. An overview of results from randomized controlled trials. JAMA 270:1589-1595

176. Hine LK, Laird N, Hewitt P, Chalmers TC (1989) Meta-analytic evidence against prophylactic use of lidocaine in acute myocardial infarction. Arch Intern Med 149:2694-2698

177. Swedberg K, Held P, Kjekshus J, Rasmussen K, Ryden L, Wedel H (1992) Effects of the early administration of enalapril on mortality in patients with acute myocardial infarction. Results of the Cooperative New Scandinavian Enalapril Survival Study II (CONSENSUS II). N Engl J Med 327:678684

178. (1995) ISIS-4: a randomised factorial trial assessing early oral captopril, oral mononitrate, and intravenous magnesium sulphate in 58,050 patients with suspected acute myocardial infarction. ISIS-4 (Fourth International Study of Infarct Survival) Collaborative Group. Lancet 345:669-685

179. (1998) Indications for ACE inhibitors in the early treatment of acute myocardial infarction: systematic overview of individual data from 100,000 patients in randomized trials. ACE Inhibitor Myocardial Infarction Collaborative Group. Circulation 97:2202-2212

180. Pfeffer MA, McMurray JJ, Velazquez EJ et al (2003) Valsartan, captopril, or both in myocardial infarction complicated by heart failure, left ventricular dysfunction, or both. N Engl J Med 349:18931906

181. Patti G, Pasceri V, Colonna G et al (2007) Atorvastatin pretreatment improves outcomes in patients with acute coronary syndromes undergoing early percutaneous coronary intervention: results of the ARMYDA-ACS randomized trial. J Am Coll Cardiol 49:1272-1278

182. Hulten E, Jackson JL, Douglas K, George S, Villines TC (2006) The effect of early, intensive statin therapy on acute coronary syndrome: a meta-analysis of randomized controlled trials. Arch Intern Med 166:1814-1821

183. Heeschen C, Hamm CW, Laufs U, Snapinn S, Bohm M, White HD (2002) Withdrawal of statins increases event rates in patients with acute coronary syndromes. Circulation 105:1446-1452 